\title{
UNE NOUVELLE ESPÈCE DE TOMETES (TELEOSTEI : CHARACIDAE : SERRASALMINAE) DU BOUCLIER GUYANAIS, TOMETES LEBAILI N. SP.
}

\author{
M. JÉGU (1), P. KEITH (2), E. BELMONT-JÉGU (3)
}

(1) Antenne IRD, Laboratoire d'Ichtyologie, M.N.H.N., 43 rue Cuvier, 75231 PARIS Cedex 05 France. E-mail : jegu@mnhn.fr

(2) Laboratoire d'Ichtyologie, M.N.H.N., 43 rue Cuvier, 75231 PARIS Cedex 05 France. E-mail : keith@mnhn.fr

(3) Chercheur attaché au Laboratoire d'Ichtyologie, M.N.H.N., 43 rue Cuvier, 75231 PARIS Cedex 05 France.

\section{RÉSUMÉ}

Tometes lebaili n. sp. diffère de Tometes trilobatus Valenciennes, 1850, par la position de la bouche, oblique et dirigée vers le haut. T. lebaili présente 7 à 8 dents à la série labiale du dentaire contre 5 chez T. trilobatus. T. trilobatus, citée des rapides de l'Araguari (Amapá, Brésil) à l'Oyapock (Guyane française), est remplacée plus à l'ouest par T. lebaili n. sp., décrite de la Mana (Guyane fr.) au Commewine (Surinam) et peut-être présente dans le Sinnamary. La taille maximale observée pour T. lebaili est de $512 \mathrm{~mm}$ LS.

Mots-clés : Tometes lebaili, espèce nouvelle, Guyane française, Surinam, Characidae, Serrasalminae.

\section{A NEW SPECIES OF TOMETES \\ (TELEOSTEI: CHARACIDAE: SERRASALMINAE)} FROM THE GUIANIAN SHIELD, TOMETES LEBAILI N. SP.

\begin{abstract}
Tometes lebaili n. sp. differs from Tometes trilobatus Valenciennes, 1850, by the upturned mouth and 7 to 8 labial dentary teeth against 5 in T. trilobatus. T. trilobatus, cited in the running waters from Araguari (Amapá, Brazil) to Oyapock Basin (French Guiana) is replaced by T. lebaili n. sp., described from la Mana (French Guiana) to Commewine basin (Surinam) and perhaps occurring in the Sinnamary Basin. The maximal size reported for T. lebaili is $512 \mathrm{~mm} \mathrm{SL}$.
\end{abstract}

Key-words : Tometes lebaili, new species, French Guiana, Surinam, Characidae, Serrasalminae. 


\section{INTRODUCTION}

VALENCIENNES (in CUVIER et VALENCIENNES, $1850: 225$ ) propose le genre Tometes pour T. trilobatus Valenciennes, 1850 dont les dents aux mâchoires sont " comparables à de véritables incisives ». Cette espèce est rapidement placée en synonymie avec $M$. setiger Müller et Troschel, 1844, espèce-type de Myleus Müller et Troschel, 1844. Tometes devient synonyme de Myleus et sera identifié par GÉRY et al. (1991 : 37-38) dans l'Oyapock comme Myleus pacu pour les jeunes et Mylesinus sp. pour un grand spécimen. A l'issue d'une description complémentaire de Myleus setiger, JÉGU et SANTOS (2002) montrent que les types de Tometes trilobatus sont différents de Myleus setiger au niveau de certains caractères méristiques et de la forme et la position des dents aux mâchoires. JÉGU et al. (sous presse) proposent la revalidation du genre Tometes après la description complémentaire de Tometes trilobatus.

Un grand Serrasalminae femelle à bouche oblique observé en 1983 au Saut Singateteï (Maroni, Guyane française) par le Dr. Le Bail (INRA, Rennes) est identifié, d'après photo, comme Mylesinus schomburgki par LE BAIL et al. (1984: 88, Figure 5a) et Mylesinus sp. par GÉRY et al. (1991 : 38). Ce même spécimen est représenté dans l'Atlas des Poissons d'eau douce de Guyane française (PLANQUETTE et al., 1996 : 364-365) et identifié comme Mylesinus sp. Les auteurs signalent que cette espèce de grande taille est aussi observée dans la Mana et le Sinnamary (?), mais KEITH et al. (2000 : 14) indiquent qu'elle appartient au genre Tometes. PAGÉZY et JÉGU (2002) font aussi état de cette nouvelle espèce de Tometes que les amérindiens Wayana du haut Maroni nomment watau yaikë.

Après avoir consulté les premiers spécimens réunis par Paul Planquette à l'INRA de Kourou, nous avons pu collecter et examiner de nouveaux spécimens issus des bassins de la Mana et du Maroni. Ce matériel présente tous les caractères de Tometes relevés par JÉGU et al. (sous presse). II s'agit d'une nouvelle espèce dont nous proposons la description et qui se différencie notamment de Tometes trilobatus par la position de la bouche, oblique orientée vers le haut.

\section{MATÉRIEL ET MÉTHODES}

Les récoltes ont d'abord été réalisées dans le cadre des inventaires faunistiques de Guyane française menés par l'INRA en 1986 puis au cours des campagnes menées par le MNHN et l'INRA sur les Conventions INRA / DIREN-GF (93192/1993) en 1995 et 1996 et INRA / DIREN-GFC (41011) en 1997. Les dernières collectes dans le Haut Maroni ont été réalisées dans le cadre des programmes SOFT 1996 (Convention IRD / ECOFOR 2350, en 1998-99, Convention MNHN / ECOFOR 048 et MNHN / DIREN 049 en 2001), Ecosystèmes Tropicaux du MATE (Conventions IRD / ECOFOR 4403.00 et IRD / CSP 2548 en 2000). Les radiographies de contact aux rayons $X$ ont été exécutées au laboratoire d'ichtyologie du MNHN à l'aide d'un appareil Faxitron 43855A sur des négatifs radio AGFA DW Structurix.

Les mesures ont été établies sur des spécimens de 92 à $460 \mathrm{~mm}$ de longueur standard (LS) et les comptages sur des spécimens de 20 à $460 \mathrm{~mm}$ de LS, issus des bassins du Maroni et de la Mana, plus un spécimen libellé du Sinnamary mais dont l'origine est incertaine (Figure 1). Les mesures ont été prises sur les radiographies ou directement sur les spécimens. Les points remarquables, entre lesquels les distances ont été mesurées au pied à coulisse suivant JÉGU et al. (1991) et FINK (1993), sont figurés dans MERCKX et al. (2000 : Figure 3a et b).

Les caractères méristiques relevés sur les radiographies sont les suivants : nombre de rayons aux nageoires dorsale, anale et caudale ; nombre de serrae abdominales ; nombre de vertèbres en avant du premier et en arrière du dernier ptérygiophore de la 
dorsale ; nombre de supraneuraux et nombre total de vertèbres. Directement sur les spécimens nous avons compté : le nombre d'écailles perforées le long de la ligne latérale avant et après l'hypural, le nombre de séries d'écailles autour du pédoncule caudal au niveau le plus étroit du pédoncule et le nombre de branchiospines sur le premier arc branchial.

La description du squelette a été réalisée d'après l'examen de squelettes secs (MNHN 2001-1237, 290 mm de LS, mâle ; MNHN 2001-1238, 300 mm de LS, femelle ; MNHN 2001-1219, $155 \mathrm{~mm}$ de LS) et complétée par l'examen des radiographies de 80 spécimens de 20 à $250 \mathrm{~mm}$ de LS ainsi que l'examen de deux spécimens diaphanisés (MNHN 2001-2677, 22-23 mm de LS). La diaphanisation a été réalisée suivant la technique décrite par TAYLOR et VAN DYKE (1985). La nomenclature française des os est celle de COURTEMANCHE et LEGENDRE (1985).

La liste du matériel de Tometes trilobatus examiné pour comparaison est celle qui figure dans JÉGU et al., sous presse.

La liste des acronymes utilisés dans le texte est la suivante :

- CSP, Conseil Supérieur de la Pêche, Paris,

- DIREN, Direction régionale de l'environnement, Cayenne, Guyane française,

- IRD, Intitut de Recherche pour le Développement, Paris,

- INRA, Institut National de la Recherche Agronomique, Kourou, Guyane française,

- IRSNB, Institut Royal des Sciences de Belgique, Bruxelles, Belgique,

- MNHN, Muséum National d'Histoire Naturelle, Paris, France, Suriname.

- NZCS, National Zoological Collection, University of Suriname, Paramaribo,

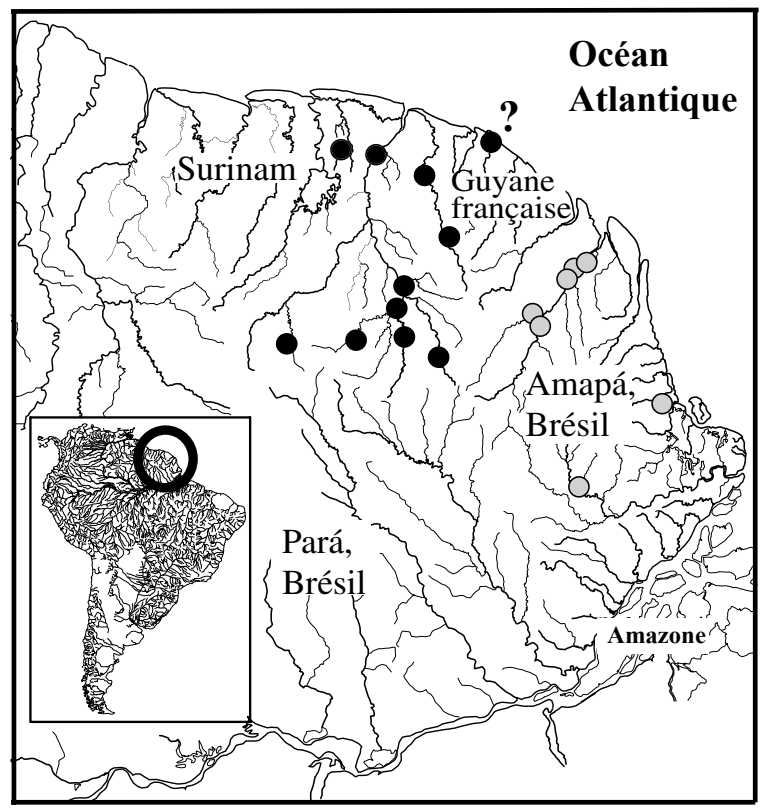

Figure 1

Tometes lebaili n. sp. : carte de répartition de Tometes lebaili (points noirs) et de T. trilobatus (points grisés).

Figure 1

Tometes lebaili $\mathrm{n}$. sp.: distribution area of Tometes lebaili (black points) and T. trilobatus (grey points). 
Tometes lebaili n. sp.

Mylesinus schomburgki (non Valenciennes). - LE BAIL et al., Bull. liaison du GRAAG, $9: 88$, Figure 5a, 1984, Maroni.

Mylesinus. - GÉRY et al., Cybium, 15 (1) : 38, 1991, Maroni.

Mylesinus ? sp. - PLANQUETTE et al., Atlas des poissons d'eau douce de Guyane, tome 1:359 (Figure 7a), 1996, Maroni (dessin).

Mylesinus ? sp. 1. - PLANQUETTE et al., Atlas des poissons d'eau douce de Guyane, tome 1 : 364-365, 1996, Saut Singateteï, Maroni (description, photographie).

Tometes n. sp. - KEITH et al., Atlas des poissons d'eau douce de Guyane, tome 2, fascicule I : 14, 2000 (citation).

Myleus tometes. - FRÉRY et al., Environmental Health Perspectives, 109 (5) : 453, 2001, Maroni (contamination au méthylmercure).

Tometes lebaili Jégu et al. - PAGÉZY et JÉGU, Bull. Fr. Pêche Piscic., 364 : 49, Figure 2b, 2002, Maroni (valeur patrimoniale).

\section{DIAGNOSE}

Dents du dentaire incisiformes bi- et tricuspides, engrenées. Prémaxillaire laminaire, avec un processus transversal. Dernières dents de la série labiale du prémaxillaire tricupsides, plus larges que hautes, couronne sigmoïde. La bouche est oblique et dirigée vers le haut, caractère unique chez les Serrasalminae présentant deux séries de dents au prémaxillaire. Toutes les dents de la série labiale du prémaxillaire sont visibles lorsque la bouche est fermée et sont dirigées vers l'extérieur de la bouche, caractères observés seulement pour les dents mammillaires chez Catoprion et chez les grands spécimens d'Acnodon normani. Le nombre de dents à la série labiale du dentaire des adultes varie de 6 à 8 à chaque demi-mâchoire. La gorge d'insertion du processus ascendant du prémaxillaire s'étend sur les $4 / 5$ du mésethmoïde.

\section{MATÉRIEL OBSERVÉ}

\section{Holotype}

MNHN 2001-2384, 108,6 mm de LS, rapides dans un bief du canal principal, en amont du village Antecume Pata $\left(03^{\circ} 18^{\prime} 06,4^{\prime \prime}\right.$ Nord et $54^{\circ} 04^{\prime} 54,1^{\prime \prime}$ Ouest), riv. Litany, fl. Maroni, coll. M. Jégu et al., oct. 2000 (voir Figure 1).

\section{Paratypes}

MNHN 2000-6047, 1 ex., 92,3 mm de LS, même localité que l'holotype. - MNHN 2000-6038, 2 ex., 126,4-147,2 mm de LS, même localité. - MNHN 2001-1231, 2 ex., 164$168 \mathrm{~mm}$ de LS, même localité. - MNHN 2001-1212, 1 ex., 144,2 mm de LS, village Antecume Pata, riv. Litany, fl. Maroni, coll. Y. Fermon et al., fev. 2001. - MNHN 2001-1213, 1 ex., $172 \mathrm{~mm}$ de LS, même localité. - MNHN 2001-1215, 1 ex., 218 mm de LS, même localité. 


\section{Matériel complémentaire}

Fleuve Mana, Guyane française : - MNHN 1998-294, 3, 110-130 mm de LS, Saut Fracas, coll. P.Y. Le Bail et al., sept. 1994. - MNHN 1998-298, 1, $275 \mathrm{~mm}$ de LS, femelle, coll. P. Planquette et al., sept 1994. - MNHN 1998-299, 1, $315 \mathrm{~mm}$ de LS, femelle, St. Fracas, coll. P. Planquette et al., sept.1994. - Collection pers. E. Vigneux, 1 tête (longueur $106 \mathrm{~mm}$ ), St. Ananas, coll. E. Vigneux., sept.1995. - Collection pers. P. Keith, 1 tête (longueur $105 \mathrm{~mm}$ ), St. Ananas, coll. P. Keith, sept. 1995. - Collection pers. P.Y. Le Bail, 1 tête (longueur 109 mm), St. Ananas, coll. P.Y. Le Bail et al., sept. 1995.

Fleuve Maroni, riv. Tapanahony, Surinam : - IRSBN 19296, 1, $415 \mathrm{~mm}$ de LS, Papadronsolea, district Marowijne, riv. Paloemeu, S.M. Leopold III et J.P. Gosse, 01 nov. 1966.

Fleuve Maroni, riv. Oulemary, Surinam : - MNHN 1998-1346, 4 ex. dont une tête,

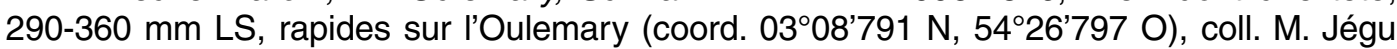
et H. Pagézy, oct. 1998.

Fleuve Maroni, riv. Tampoc, Guyane française : - MNHN 2000-6488, 1 ex., 20,7 mm de LS, Saut Pierkuru, Jégu et al., oct. 2000.

Fleuve Maroni, riv. Litany, rapides près du village Antecume Pata, Guyane française : - MNHN 1998-1347, 11 ex., 20-73 mm de LS, coord. 03¹7’757 N 540.'219 O, coll. M. Jégu, oct. 1998. - MNHN 1999-626, 1 ex., coll. M. Jégu et H. Pagézy, mai 1999. - MNHN 1999-641, 1 ex., 290 mm de LS, male, coll. M. Jégu et H. Pagézy, mai 1999. - MNHN 20011206, 1 ex., 315 mm de LS, coll. Y. Fermon et al., juin 2001. - MNHN 2001-1207, 1 ex., $266 \mathrm{~mm}$ de LS, Fermon et al., juin 2001. - MNHN 2001-1208, 1 ex., $373 \mathrm{~mm}$ de LS, coll. Y. Fermon et al., juin 2001. - MNHN 2001-1216, 1 ex., 249 mm de LS, coll. Y. Fermon et al., juin 2001. - MNHN 2001-1217, 1 ex., 328 mm de LS, coll. Y. Fermon et al., juin 2001. MNHN 2001-1218, 1 ex., 328 mm de LS, coll. Y. Fermon et al., juin 2001. - MNHN 20011220, 1 ex., 369 mm de LS, coll. Y. Fermon et al., juin 2001. - MNHN 2001-1209, 1 ex., 95 mm de LS, coll. Y. Fermon et al., juin 2001. - MNHN 2001-1210, 1 ex., 99 mm de LS coll. Y. Fermon et al., juin 2001. - MNHN 2001-1211, 1 ex., $124 \mathrm{~mm}$ de LS, coll. Y. Fermon et al., juin 2001. - MNHN 2001-1214, 1 ex., 224 mm de LS coll. Y. Fermon et al., juin 2001. MNHN 2001-1219, 1 ex., 163 mm de LS, coll. Y. Fermon et al., juin 2001. - MNHN 20011221, 1 ex., 390 mm de LS, coll. Y. Fermon et al., juin 2001. - MNHN 2001-1222, 1 ex., $416 \mathrm{~mm}$ de LS, coll. Y. Fermon et al., juin 2001. - MNHN 2001-1223, 1 ex., $512 \mathrm{~mm}$ de LS, coll. Y. Fermon et al., juin 2001. - MNHN 2001-1228, 1 ex., 329 mm de LS, coll. Y. Fermon et al., juin 2001. - MNHN 2001-1229, 1 ex., 430 mm de LS, coll. Y. Fermon et al., juin 2001. - MNHN 2001-2383, 1 ex., 33,2 mm de LS, rapides dans un bief du canal principal, amont du village $\left(03^{\circ} 18^{\prime} 06,4^{\prime \prime} \mathrm{N}\right.$ et $\left.54^{\circ} 04^{\prime} 54,1^{\prime \prime} \mathrm{O}\right)$, coll. Jégu et al., oct. 2000. - MNHN 2000-6067, 1 ex., aval du village, coll. Jégu et al., oct. 2000. - MNHN 2000-6097, 3 ex., 28,3-33,3 mm de LS, rapides dans un bief du canal principal, amont du village $\left(03^{\circ} 18,06,4^{\prime \prime} \mathrm{N}\right.$ et $54^{\circ} 04^{\prime} 54,1$ " O), coll. Jégu et al., oct. 2000. - MNHN 2000-6188, 26 ex., 21,8-51,6 mm de LS, rapides en aval du village, coll. Jégu et al., oct. 2000. - MNHN 2001-2677, 2 ex. diaphanisés, 22-23 mm LS, rapides en aval du village, coll. Jégu et al., oct. 2000. - MNHN 2000-6204, 6 ex., 33,7-49,0 mm de LS, rapides dans un bief du canal principal, amont du village $\left(03^{\circ} 18^{\prime} 06,4^{\prime \prime} N\right.$ et $54^{\circ} 04^{\prime} 54,1^{\prime \prime}$ O), coll. Jégu et al., oct. 2000. - MNHN 2000-6221, 1 ex., rapides en aval du village, coll. Jégu et al., oct. 2000. - MNHN 2000-6234, 3 ex., $30,7-32,5 \mathrm{~mm}$ de LS, rapides dans un bief du canal principal, aval village $\left(03^{\circ} 18^{\prime} 00,2^{\prime \prime} \mathrm{N}\right.$ et $54^{\circ} 03^{\prime} 50,6^{\prime \prime}$ O), coll. Jégu et al., oct. 2000. - MNHN 2000-6295, 3 ex., 30,4-40,3 mm de LS, rapides dans un bief du canal principal, amont village $\left(03^{\circ} 18^{\prime} 06,4^{\prime \prime} \mathrm{N}\right.$ et $\left.54^{\circ} 04^{\prime} 54,1^{\prime \prime} \mathrm{O}\right)$, coll. Jégu et al., oct. 2000. - MNHN 2000-6351, 1 ex., 22,7 mm de LS, Antecume Pata, coll. Jégu et al., oct. 2000. - MNHN 2000-6402, 2 ex., 23,7-25,2 mm de LS, Antecume Pata, coll. Jégu et al., oct. 2000. - MNHN 2000-6406, 2 ex., rapides dans un bief du canal 
principal, aval village $\left(03^{\circ} 18^{\prime} 00,2^{\prime \prime} \mathrm{N}\right.$ et $\left.54^{\circ} 03^{\prime} 50,6^{\prime \prime} \mathrm{O}\right)$, coll. Jégu et al., oct. 2000. - MNHN 2000-6436, 2 ex., rapides dans un bief du canal principal, amont village $\left(03^{\circ} 18^{\prime} 06,4^{\prime \prime} \mathrm{N}\right.$ et $54^{\circ} 04^{\prime} 54,1^{\prime \prime}$ O), coll. Jégu et al., oct. 2000. - MNHN 2000-6449, 1 ex., 39,1 mm de LS, rapides dans un bief du canal principal, amont du village $\left(03^{\circ} 18^{\prime} 06,4^{\prime \prime} \mathrm{N}\right.$ et $\left.54^{\circ} 04^{\prime} 54,1^{\prime \prime} \mathrm{O}\right)$, coll. Jégu et al., oct. 2000. - MNHN 2000-6500, 11 ex., 20,6-33,5 mm de LS, rapides dans

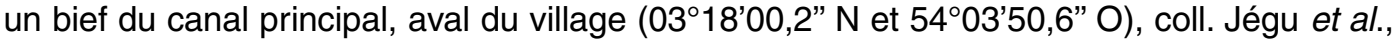
oct. 2000. - MNHN 2001-1237, squelette de mâchoire, 290 mm de LS, mâle, région Antécume Pata, coll. Jégu et Pagézy, juin 1999. - MNHN 2001-1238, squelette entier, $300 \mathrm{~mm}$ de LS, femelle, région Antécume Pata, coll. Jégu et Pagézy, juin 1999.

Fleuve Maroni, région de Maripasoula (marché ?), Guyane française ou Surinam : MNHN 1993-3450, 1, 415 mm de LS, femelle, coll. P. Planquette, mars 1996. - MNHN 1993-3451, 1, 390 mm de LS, mâle, coll. P. Planquette, mars 1996. - MNHN 1993-3452, 1, $268 \mathrm{~mm}$ de LS, femelle, coll. P. Planquette, mars 1996. - MNHN 1993-3453, 1, 202 mm de LS, femelle, coll. P. Planquette, mars 1996.

Fleuve Sinnamary (?), Guyane française : - MHNH 1998-304, 1, 460 mm de LS, mâle, Pointe Combi, Domaine Combi-Cirad, Sinnamary, coll. Ph. Lachenaud (origine sujette à caution, voir discussion).

Guyane Française, Fl. Mana ou Maroni (prov. ind.): - MNHN 1998-305, 1 ex, 410 mm de LS, mâle. 1998-99.

Surinam, Commewine River, Mapaná Creek : - MZCS non cat., 1 ex., coll. Ouboter,

\section{DESCRIPTION}

\section{Morphologie}

Les principaux caractères morphométriques sont présentés en pourcentage de la longueur standard au Tableau I. Nous verrons plus bas que plusieurs caractères morphométriques sont marqués par une nette allométrie bien que l'intervalle de tailles considérées (90-460 mm de LS) ne prenne pas en compte les juvéniles qui présentent de fortes allométries dans le genre Tometes (JÉGU et al., sous presse).

Le corps est losangique à ovoïde, même chez les plus petits spécimens observés (Figures 2 et 3). Le profil du museau est rectiligne et la concavité au-dessus des yeux, peu marquée chez les plus grands spécimens, est à peine perceptible chez les plus jeunes. Le profil prédorsal est rectiligne chez les spécimens de moins de $100 \mathrm{~mm}$ LS et régulièrement courbé jusqu'à l'origine de la dorsale chez les autres. Le profil ventral est courbé jusqu'en avant des pelviennes, puis rectiligne jusque l'origine de l'anale chez les spécimens de moins de $150 \mathrm{~mm}$ LS. Chez ceux de plus de $300 \mathrm{~mm} \mathrm{LS}$, le profil ventral est sub-rectiligne en avant des pelviennes. L'insertion du premier rayon de l'anale est situé à la verticale des derniers rayons de la dorsale. La base de la nageoire anale est très oblique par rapport à l'axe longitudinal du corps. La hauteur du corps présente une allométrie positive chez les jeunes spécimens pour atteindre un maximum de 60-66 \% de la LS vers 150-200 mm de LS puis décroître régulièrement jusqu'à 50 \% de la LS pour les plus grands spécimens. Aucune différence de hauteur du corps liée au sexe n'a pu être mise en évidence.

La tête est allongée et triangulaire car le museau est sub-rectiligne depuis le bord antérieur du prémaxillaire. L'espace interorbitaire présente une forte allométrie positive, variant de $10 \%$ de la LS chez les plus jeunes à $16 \%$ chez les plus grands spécimens. 
Le bord inférieur de l'œil est situé au niveau de la commissure de la bouche, mais l'œil, nettement postérieur à la commissure est situé légèrement en arrière du milieu de la tête. Le diamètre de l'œil présente une croissance isométrique à la LS alors que la longueur du museau (6-10 \% de la LS) présente une allométrie légèrement minorante.

\section{Tableau I}

Variation des caractères morphométriques chez Tometes lebaili n. sp. (Int. Var. = intervalle de variation ; Moy = moyenne $;$ ET = Ecart-type).

\section{Table I}

Variation of morphometric characters in Tometes lebaili n. sp. (Int. Var. = variation interval; Moy = mean; ET = Standard deviation).

\begin{tabular}{|c|c|c|c|c|c|c|c|}
\hline & \multirow{2}{*}{ Holotype } & \multicolumn{3}{|c|}{ Paratypes (8 ex.) } & \multicolumn{3}{|c|}{ Mat. complémentaire (11 ex.) } \\
\hline & & Int. var. & Moy. & ET & Int. var. & Moy. & ET \\
\hline Longueur standard (mm) & 108,6 & $92,3-218$ & 154,0 & 36,7 & $109,8-460$ & 281,2 & 129,4 \\
\hline Hauteur du corps & 59,3 & $58,5-66,9$ & 62,3 & 2,7 & $50,0-63,9$ & 57,6 & 4,0 \\
\hline Longueur de la tête & 27,5 & $26,6-29,7$ & 28,0 & 1,0 & $25,3-28,9$ & 27,2 & 1,2 \\
\hline Distance interorbitaire & 10,3 & $10,2-13,7$ & 12,5 & 1,2 & $11,1-16,0$ & 13,6 & 1,6 \\
\hline Diamètre vertical de l'œil & 9,8 & $8,4-10,1$ & 9,3 & 0,5 & $6,8-9,7$ & 7,9 & 0,9 \\
\hline Longueur du museau & 9.0 & $9,2-11,7$ & 10,3 & 0,8 & $8,3-11,6$ & 9,9 & 0,9 \\
\hline Largeur de I'IO3 & 2,6 & $2,6-3,5$ & 2,9 & 0,3 & $2,6-3,8$ & 2,9 & 0,3 \\
\hline Espace nu à la joue & 4,2 & $3,6-4,5$ & 4,0 & 0,3 & $3,0-4,8$ & 3,7 & 0,5 \\
\hline Largeur de I'IO4 & 3,9 & $3,5-4,0$ & 3,7 & 0,2 & $2,8-4,0$ & 3,5 & 0,4 \\
\hline Distance postorbitaire & 9,5 & $8,6-10,7$ & 9,3 & 0,7 & $8,2-10,2$ & 9,5 & 0,7 \\
\hline Distance postoccipitale & 31,3 & $30,3-33,7$ & 32,0 & 1,3 & $29,2-31,7$ & 30,4 & 1,2 \\
\hline Distance prédorsale & 53,7 & $53,8-62,8$ & 58,9 & 2,8 & $53,5-60,2$ & 56,4 & 2,2 \\
\hline Base de la dorsale & 30,2 & $27,0-30,6$ & 29,2 & 1,1 & $27,8-33,7$ & 30,6 & 1,8 \\
\hline Distance interdorsale & 11,5 & $9,6-12,1$ & 11,1 & 0,8 & $8,4-11,8$ & 10,1 & 0,9 \\
\hline Base de l'adipeuse & 3,8 & $3,4-4,5$ & 4,0 & 0,4 & $3,1-4,6$ & 3,8 & 0,4 \\
\hline Hauteur du pédoncule & 11,6 & $10,6-12,9$ & 11,8 & 0,7 & $10,7-12,8$ & 11,7 & 0,7 \\
\hline Base de l'anale & 33,4 & $32,2-35,5$ & 33,6 & 1,1 & $31,2-35,2$ & 33,0 & 1,5 \\
\hline Distance préanale & 75,1 & $77,4-82,3$ & 79,5 & 1,8 & $75,6-86,7$ & 79,3 & 3,3 \\
\hline Distance prépectorale & 26,2 & $26,0-28,1$ & 27,1 & 0,7 & $24,6-30,3$ & 26,7 & 1,8 \\
\hline Distance prépelvienne & 59,4 & $59,5-64,7$ & 61,1 & 1,7 & $55,4-62,5$ & 59,3 & 2,6 \\
\hline Distance pelvienne-pectorale & 34,2 & $32,9-37,6$ & 34,9 & 1,4 & $29,2-37,5$ & 33,9 & 2,2 \\
\hline Distance anale-pelvienne & 18,0 & $21,0-24,4$ & 22,4 & 1,1 & $19,6-26,2$ & 23,0 & 1,8 \\
\hline Distance début dorsale-début anale & 61,0 & $60,1-69,1$ & 64,7 & 3,1 & * & * & * \\
\hline Distance fin dorsale-début anale & 49,4 & $45,0-52,7$ & 49,1 & 3,0 & * & * & * \\
\hline Distance fin dorsale-fin anale & 24,0 & $23,2-27,0$ & 25,1 & 1,4 & * & * & * \\
\hline Longueur du lobe antérieur dorsale & 26,2 & $22,8-25,9$ & 24,2 & 1,1 & $17,9-24,4$ & 21,8 & 1,9 \\
\hline Longueur des pectorales & 23,8 & $21,7-25,2$ & 23,4 & 1,0 & $19,5-25,2$ & 21,8 & 1,7 \\
\hline Longueur des pelviennes & 16,1 & $14,1-17,2$ & 15,6 & 1,0 & $12,3-18,9$ & 15,6 & 2,0 \\
\hline Longueur du lobe antérieur anal & 26,4 & $21,7-28,0$ & 24,9 & 2,0 & $17,0-26,4$ & 21,5 & 2,5 \\
\hline Longeur du second lobe anal & * & * & * & * & $15,3-23,9$ & * & * \\
\hline Largeur de la tête & 15,5 & $14,7-16,4$ & 15,5 & 0,7 & $15,0-17,7$ & 16,4 & 0,9 \\
\hline Largeur de la mâchoire inférieure & * & $7,8-9,3$ & 8,4 & 0,6 & $6,4-11,8$ & 9,1 & 1,9 \\
\hline Profondeur de la mâchoire inférieure & * & * & * & * & $6,2-11,8$ & 8,3 & 1,7 \\
\hline
\end{tabular}




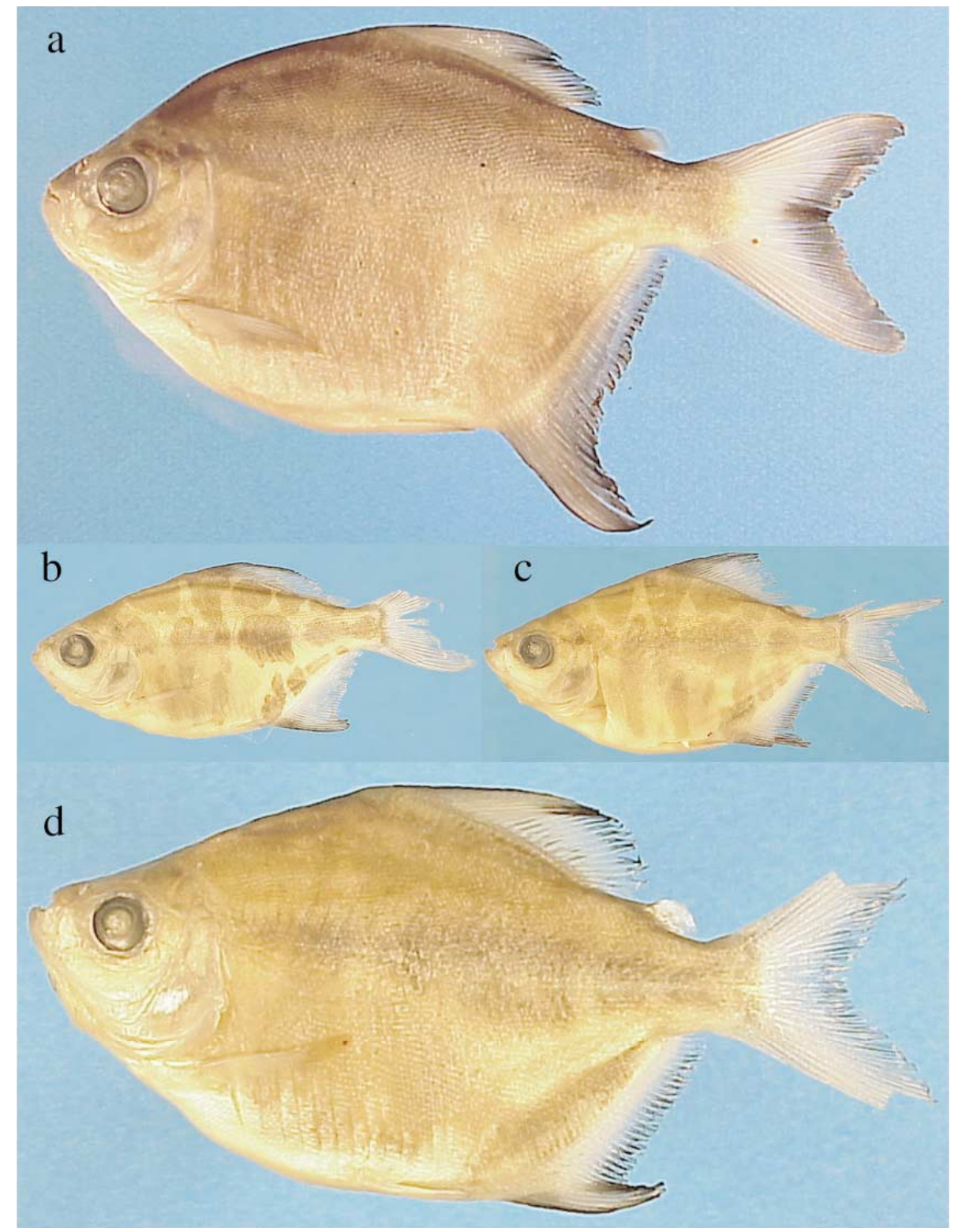

\section{Figure 2}

Tometes lebaili n. sp. : (a) holotype, MNHN 2001-2384, 108,6 mm de LS ; MNHN 2000-6188, juvéniles, (b) $23 \mathrm{~mm}$ de LS, (c) $35 \mathrm{~mm}$ de LS et (d) $53 \mathrm{~mm}$ de LS.

\section{Figure 2}

Tometes lebaili n. sp.: (a) holotype, MNHN 2001-2384, $108.6 \mathrm{~mm}$ SL; MNHN 20006188, juveniles, (b) $23 \mathrm{~mm} \mathrm{SL}$, (c) $35 \mathrm{~mm} \mathrm{SL}$ and (d) $53 \mathrm{~mm} \mathrm{SL}$.

La bouche est largement fendue, oblique et dirigée vers le haut. Toutes les dents de la série labiale du prémaxillaire sont dirigées vers l'extérieur et visibles lorsque la bouche est fermée. Cette disposition est assez proche de l'aspect agressif présent chez les carnivores Serrasalmus et le lépidophage Catoprion. Les lèvres sont étroites et le bord distal de la membrane à la commissure est lisse. Nous n'avons pas observé de lobe charnu à l'intérieur de la bouche, en arrière des séries du dentaire. 


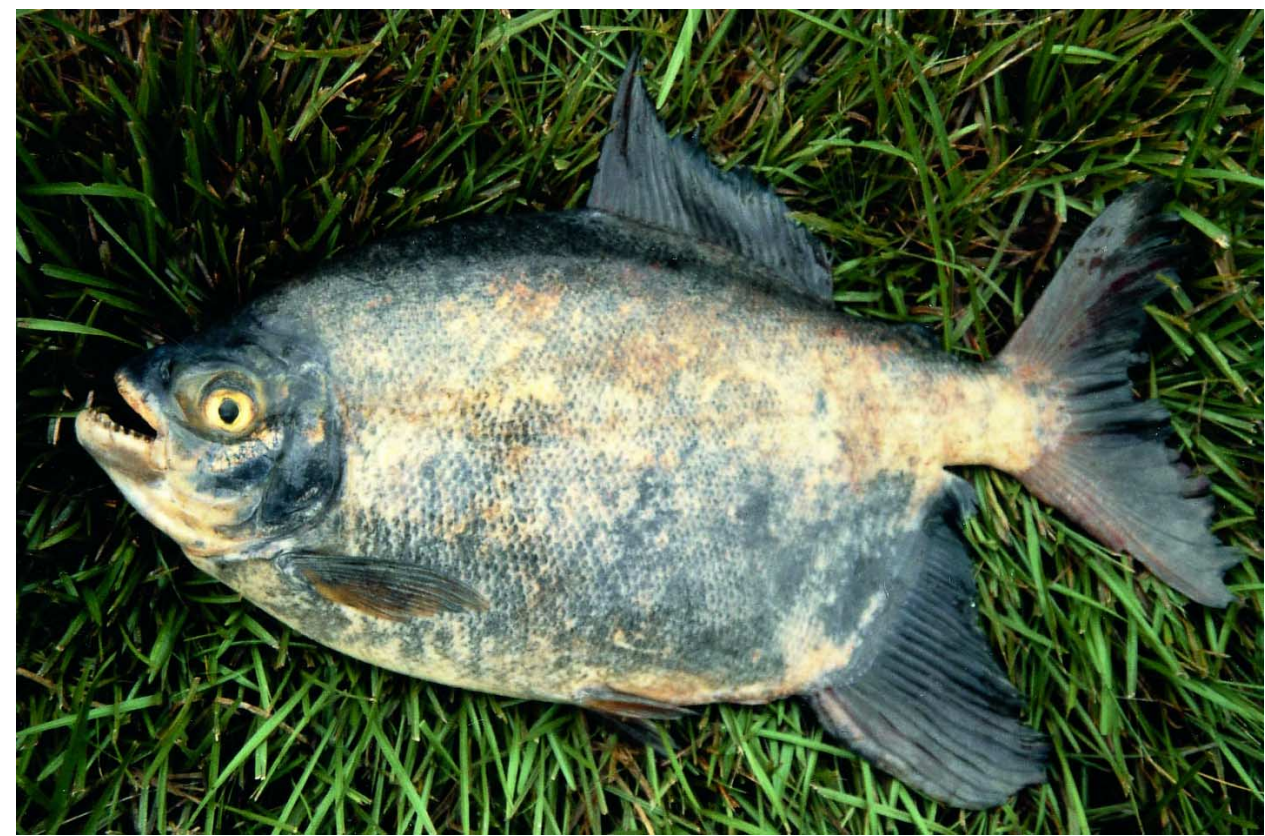

Figure 3

Tometes lebaili n. sp., femelle adulte in vivo, environ $400 \mathrm{~mm}$ de LS, Saut Singateteï, fl. Maroni (in PLANQUETTE et al., 1996).

\section{Figure 3}

Tometes lebaili n. sp., adult female in vivo, about $400 \mathrm{~mm} \mathrm{SL}$, Saut Singateteï, Maroni Riv. (in PLANQUETTE et al., 1996).

Au prémaxillaire nous avons observé 5 à 6 dents à la série labiale et 2 dents à la série linguale (Figure 4c). Les dents de la série linguale sont accolées à celles de la série labiale. Les dents des deux paires symphysaires de la série labiale ne sont pas en contact latéral. Ces dents sont incisiformes unilobées, à base conique (Figure 4a). L'apex de la couronne est plus anguleuse chez les spécimens de moins de $300 \mathrm{~mm}$ de LS et arrondi chez les autres (Figure 4b). La base des dents labiales de la troisième paire est plus allongée. Chez ces trois paires de dents, la face concave est linguale et la couronne située sur un même plan. Les 2 ou trois dents suivantes sont moins hautes, plus allongées et leur couronne est sigmoïde. Chez les plus jeunes, la couronne présente deux lobes, un médian et un antérieur, alors que chez les spécimens de plus de $300 \mathrm{~mm} \mathrm{LS}$, un troisième lobe postérieur apparaît. Les dents prennent ainsi une apparence tricuspide avec la face concave labiale. La couronne des dents de la première paire de la série linguale forme un « $\mathrm{V}$ » fermé, avec un lobe arrondi à la pointe du « V » et un second à l'extrémité distale. La couronne des dents de la deuxième paire forme aussi un « $\mathrm{V}$ », beaucoup plus ouvert avec un seul lobe dont l'apex est anguleux. Les dents de la série linguale sont plus hautes que celles de la série labiale. Chez les spécimens de moins de $50 \mathrm{~mm}$ de LS, les trois premières dents de la série labiale sont incisiformes, la concavité étant tournée vers l'arrière tandis que la couronne des dents de la série linguale et des deux dernières dents de la série labiale forme un « V » orienté vers l'extérieur de la bouche. Chez les spécimens de moins de $20 \mathrm{~mm}$ de LS, les 3 premières dents labiales sont sub-caniniformes tandis que la couronne des autres dents présente une forme de « V . 


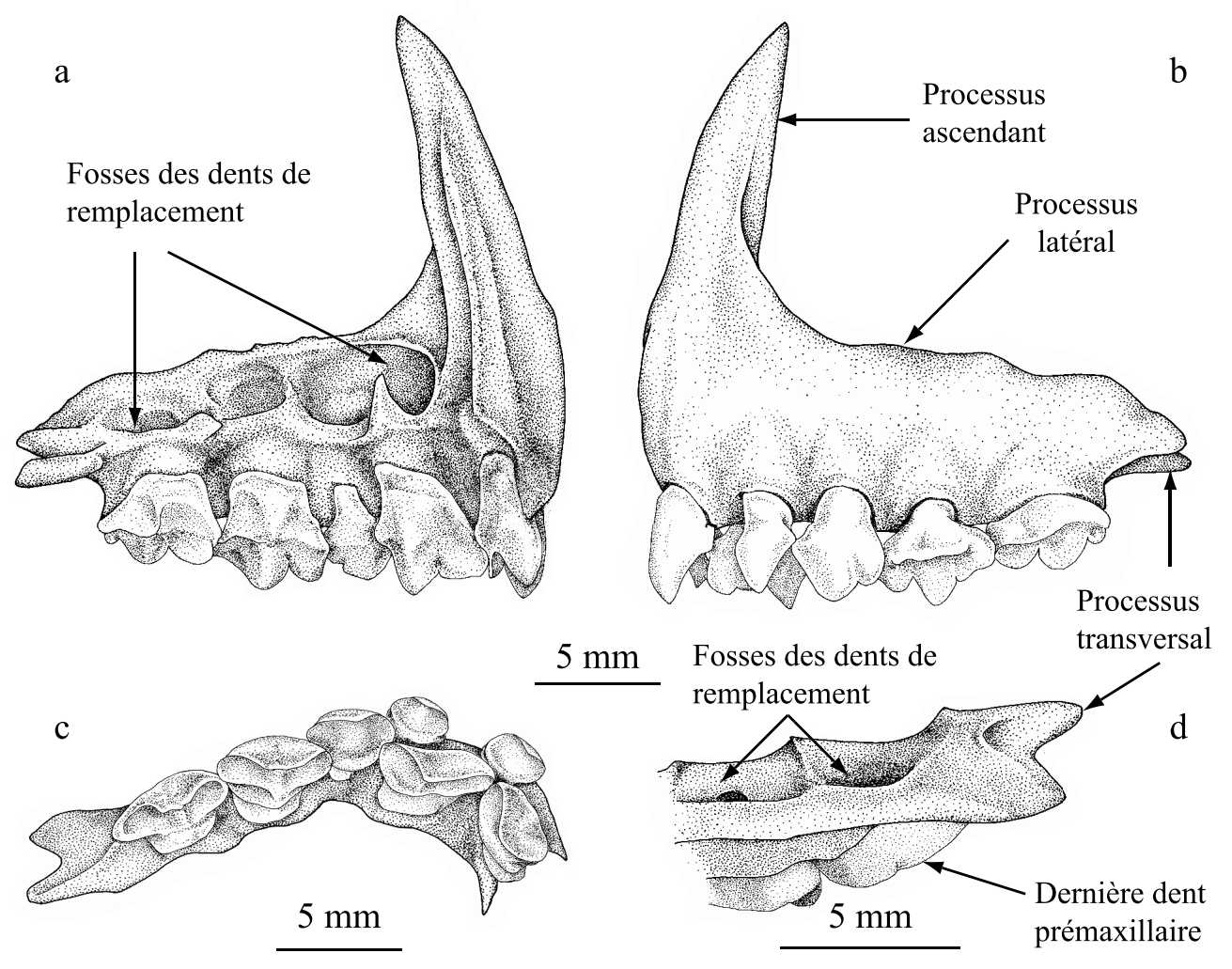

\section{Figure 4}

Tometes lebaili $\mathrm{n}$. sp., MNHN 2001-1238, $300 \mathrm{~mm}$ de LS, mâle : prémaxillaire gauche en (a) vue labiale, (b) vue linguale et (c) vue ventrale ; (d) détail du processus transversal du prémaxillaire en vue dorsale.

\section{Figure 4}

Tometes lebaili n. sp., MNHN 2001-1238, $300 \mathrm{~mm}$ SL, male: left premaxillary in (a) labial view, (b) lingual view and (c) ventral view; (d) detail of the premaxillary transversal process in dorsal view.

Au dentaire, on compte 7 à 8 dents incisiformes à la série labiale et une paire de dents symphysaires linguales coniques, fines, hautes et pointues (Figure $5 \mathrm{c}$ ). Les dents de la série labiale sont bicuspides, tricuspides pour la paire médiane (Figure 5a). La couronne de la dent est située sur un plan antéro-postérieur, sauf pour les dents médianes (Figure $5 \mathrm{~d}$ ). Le lobe antérieur, placé en position médiane de la dent, est fin, haut et son axe est légèrement incliné vers l'arrière (Figure $5 b$ et $d$ ), comme on peut l'observer chez Serrasalmus. L'apex forme un angle aigu et pointu chez les plus petits spécimens, plus arrondi et en pointe émoussée chez les autres. Le lobe postérieur, très réduit et latéral, est enclavé dans une gorge de la dent immédiatement postérieure. La couronne de toutes les dents est brun foncé alors que la base est blanche. Jusqu'à $50 \mathrm{~mm}$ de LS, le nombre de dents à la série labiale du dentaire est réduit à 5 . II s'agit de dents dont la cuspide principale est terminée en une pointe. Chez les spécimens de moins de $20 \mathrm{~mm}$ de LS, les dents sont sub-caniniformes.

L'insertion du premier rayon de la dorsale est situé au milieu du corps et la distance prédorsale ne présente pas d'allométrie dans l'intervalle de taille étudié. Les rayons sont toujours plus courts que la longueur de la tête. Les spécimens de 100 à $130 \mathrm{~mm}$ de LS présentent un petit lobe antérieur, mais le bord distal de la nageoire est rectiligne chez les autres. Chez les mâles de $450 \mathrm{~mm}$ de LS, nous avons pu observer une différentiation des 
rayons en longs filaments. La distance dorsale/adipeuse représente environ $1 / 3$ de la base de la dorsale. La nageoire adipeuse est très courte (moins d'un sixième de la base de la dorsale). Le bord distal de la nageoire est sub-rectiligne.

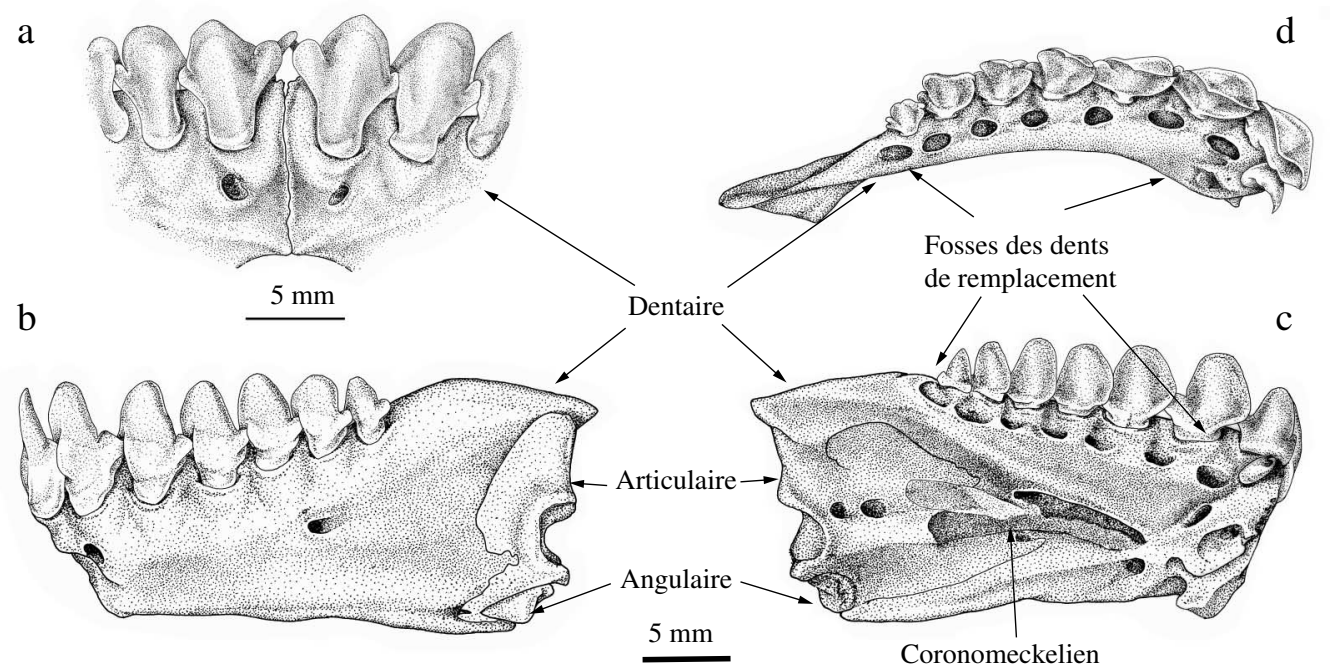

\section{Figure 5}

Tometes lebaili n. sp., MNHN 2001-1238, $300 \mathrm{~mm}$ de LS, mâle : (a) dentaires en vue frontale ; dentaire gauche en (b) vue linguale, (c) vue labiale et (d) vue dorsale.

\section{Figure 5}

Tometes lebaili n. sp., MNHN 2001-1238, $300 \mathrm{~mm}$ SL, male: (a) lower maxilla in frontal view; left dentary in (b) lingual view, (c) labial view and (d) dorsal view.

Chez les plus petits spécimens le bord distal de la nageoire anal est falciforme, les premiers rayons formant un lobe étroit et allongé. Chez les spécimens de plus de $300 \mathrm{~mm}$ de LS, ce lobe est plus court et arrondi. Chez les mâles de plus de $300 \mathrm{~mm}$ de LS, un second lobe anal, plus pointu et plus allongé, est centré sur les $14^{\text {ème }}$ et $15^{\text {teme }}$ rayons branchus. Au niveau de ce lobe, les rayons sont soudés en une tige rigide. Chez les spécimens de plus de $450 \mathrm{~mm}$ de LS, l'extrémité de certains rayons est différenciée en un double crochet du même type que celui observé chez Tometes trilobatus (JÉGU et al., sous presse) et Mylesinus paraschomburgkii (JÉGU et al., 1989).

Les pectorales sont courtes et leur pointe atteint à peine la verticale à l'origine de la dorsale. Les pelviennes sont encore plus courtes. Leur insertion est située à la verticale du premier quart de la dorsale et leur pointe n'atteint pas l'origine de l'anale.

Dans la cavité abdominale, l'intestin est enroulé suivant le même schéma que celui observé chez Mylesinus paraschomburgkii (JÉGU et al., 1989 : Figure 12). L'allongement de l'intestin présente une allométrie positive, comme chez les autres Serrasalminae phytophages (LEITE et JÉGU, 1990 : Figure 3). Chez des spécimens de 250 à $580 \mathrm{~mm}$ de LS, la longueur de l'intestin varie de 100 à plus de $500 \%$ de la LS (Figure 6a).

Chez les adultes, la chambre postérieure de la vessie natatoire est conique et courbe (Figure 7a), prolongée par un court diverticule à l'extrémité postérieure (longueur 20 à $33 \%$ de la LS). La chambre antérieure est ovale. Sa longueur varie de 55 à $65 \%$ de la longueur de la chambre postérieure et sa hauteur est d'environ $50 \%$ de sa longueur. Les deux chambres sont reliées par un canal enroulé en spirale et situé en position 
ventrale. Un muscle intrinsèque parcourt le côté de la seconde chambre (hauteur 15 à $20 \%$ de la hauteur de la chambre), depuis la pointe distale jusqu'au tiers antérieur de la chambre où il se ramifie en deux branches d'égale largeur. La branche dorsale atteint la partie dorsale de la paroi antérieure, la branche ventrale atteint la partie antéro-ventrale. Vers l'avant, les deux branches s'unissent respectivement avec leur homologue du coté opposé et une bande musculaire frontale située sur la partie médiane de la paroi antérieure relie les bandes dorsales et ventrales (Figure 7b). La paroi antérieure de la seconde chambre est lisse. La première chambre est dépourvue de muscle intrinsèque.
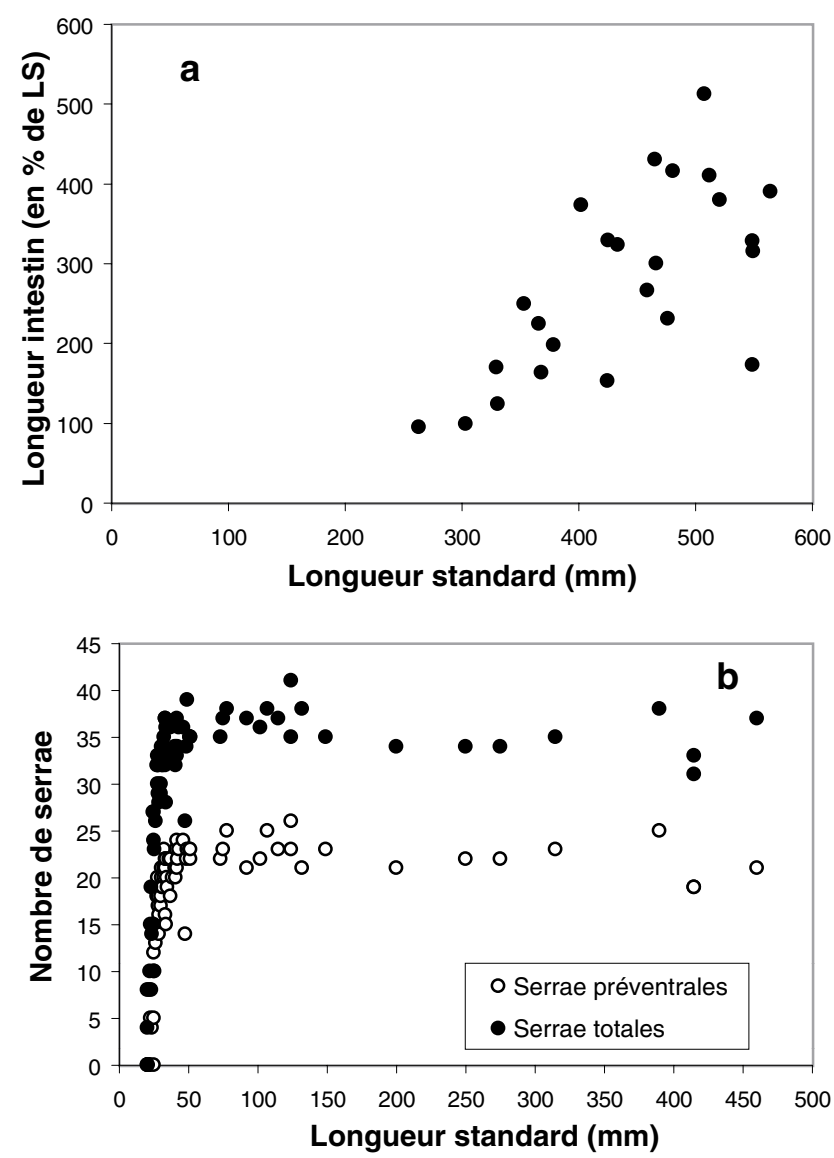

\section{Figure 6}

Tometes lebaili n. sp. : (a) variation de la longueur de l'intestin et (b) du nombre de serrae en fonction de la longueur standard.

\section{Figure 6}

Tometes lebaili $\mathrm{n}$. sp.: (a) variation of intestinal length and (b) ventral serrae number expressed in function of standard length.

Sur les flancs et au niveau de la partie antéro-dorsale de la première chambre de la vessie natatoire, un hiatus musculaire, en partie comblé par des corps graisseux, s'étend de part et d'autre des côtes pleurales 5 et 6 pour l'obliquus superioris. Pour l'obliquus inferioris, cette lacune est réduite à l'aire limitée par l'angle de la côte 5 et de la vertèbre 6.

L'adductor mandibularis A1 latéral couvre la moitié de la joue sous l'œil et laisse place à l'adductor mandibularis A2 latéral en arrière de l'œil (Figure 8). Le dilatator operculi est en forme de lame en $\mathrm{S}$. Son insertion dans la fosse dilatatoire est limitée vers l'arrière 
au niveau du pilier osseux de l'épine sphénotique pour laisser place au levator arcus operculi dont l'insertion vers l'avant se limite au bord ventral de l'épine sphénotique.

La musculature de l'œil est remarquable par la largeur du muscle droit externe, deux fois plus large que les autres muscles droits.

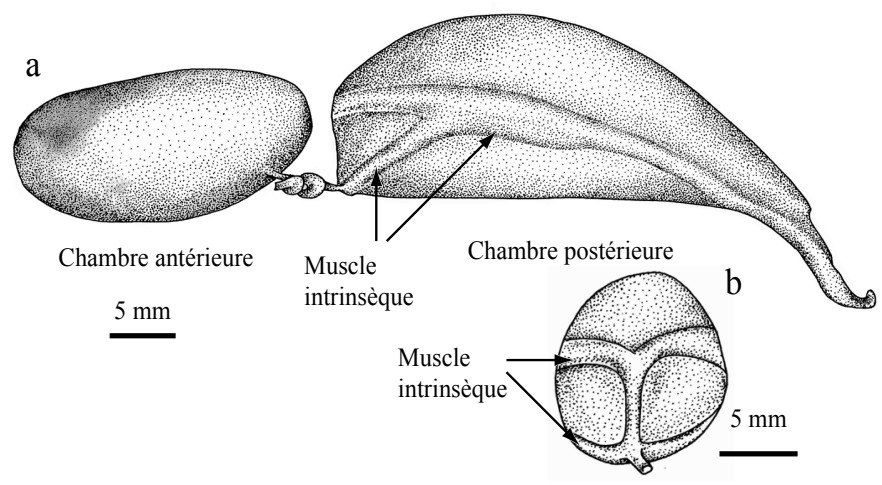

Figure 7

Tometes lebaili n. sp., MNHN 2001-1219, $155 \mathrm{~mm}$ de LS : (a) vue latérale droite de la vessie natatoire et (b) vue frontale de la seconde chambre.

\section{Figure 7}

Tometes lebaili $\mathrm{n}$. sp., MNHN 2001-1219, $155 \mathrm{~mm}$ SL: (a) right lateral view of the swimbladder and (b) frontal view of the second chamber.

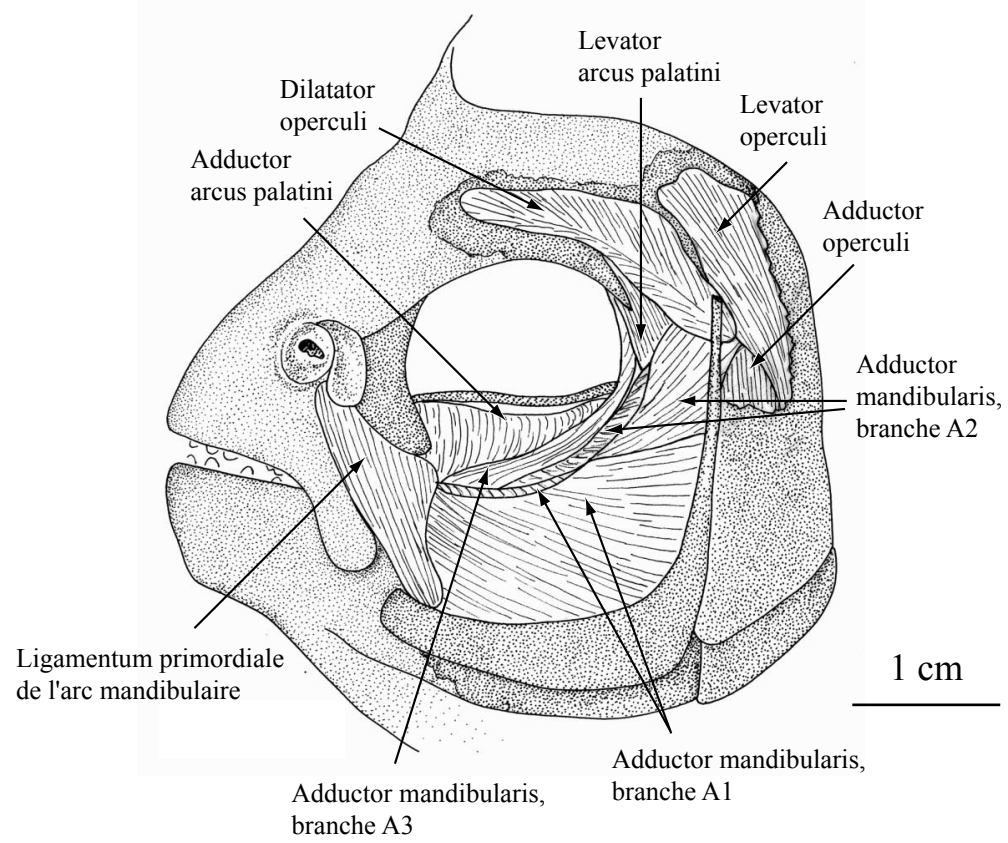

Figure 8

Tometes lebaili n. sp., MNHN 2001-1219, $155 \mathrm{~mm}$ de LS : musculature de la face gauche.

Figure 8

Tometes lebaili n. sp., MNHN 2001-1219, 155 mm SL: left facial musculature. 


\section{Caractères méristiques}

Les principaux caractères méristiques sont présentés au Tableau II. Le nombre d'écailles en ligne latérale à l'hypural varie de 67 à 72 et de 72 à 74 jusqu'à la fin de la ligne latérale. On compte 32 à 36 séries d'écailles autour du pédoncule caudal. Le nombre de rayons branchus aux nageoires varie de 29 à 33 à l'anale, de 19 à 22 à la dorsale et de 15 à 16 aux pectorales.

\section{Tableau II}

Variation des caractères méristiques chez Tometes lebaili n. sp.

\section{Table II}

Variation of meristic characters in Tometes lebaili $\mathrm{n} . \mathbf{s p}$.

\begin{tabular}{|c|c|c|c|c|c|c|}
\hline & \multirow{2}{*}{ Holotype } & \multirow{2}{*}{$\begin{array}{l}\text { Paratypes (8 ex.) } \\
\text { Intervalle variation }\end{array}$} & \multicolumn{4}{|c|}{ Spécimens complémentaires } \\
\hline & & & $\mathrm{n}$ & Intervalle variation & $\mathrm{n}$ & Intervalle variation \\
\hline Longueur standard (mm) & 108,6 & $92,3-218$ & 54 & $20-49$ & 23 & $50-460$ \\
\hline $\begin{array}{l}\text { Écailles en ligne latérale à } \\
\text { l'hypural (total) }\end{array}$ & $69(75)$ & $69-76(72-81)$ & * & * & 22 & $67-72(72-79)$ \\
\hline Écailles au pédoncule & 34 & $32-34$ & * & * & 11 & $32-36$ \\
\hline Écailles à la base anale & 6 & $6-8$ & * & * & * & * \\
\hline Rayons à l'anale & iv +29 & iv $+29-33$ & 40 & iv $+29-32$ & 13 & iv $+29-33$ \\
\hline Rayons à la dorsale & $\mathrm{ii}+20$ & $\mathrm{ii}+19-21$ & 40 & ii-iii $+19-23$ & 13 & $\mathrm{iii}+19-22$ \\
\hline Rayons aux pectorales & $\mathrm{i}+16$ & $i+16-17$ & * & * & 7 & $i+15-17$ \\
\hline Serrae prépelviennes & 23 & $20-24$ & 54 & $0-24$ & 22 & $21-26$ \\
\hline Serrae postpelviennes & 13 & $11-14$ & 54 & $0-16$ & 22 & $12-17$ \\
\hline Serrae totales & 36 & $34-37$ & 54 & $0-39$ & 22 & $35-41$ \\
\hline Dents au prémaxillaire & $5+\mathrm{ii}$ & $5+\mathrm{ii}$ & 10 & $5+\mathrm{ii}$ & 14 & $5-6+i i$ \\
\hline Dents au dentaire & $6+i$ & $6-7+i$ & 10 & $5+1$ & 13 & $6-8+i$ \\
\hline Branschiospines & $16 / 1 / 15$ & $17 / 1 / 15-16$ & ${ }^{*}$ & * & 6 & 13-16/1/16-18 \\
\hline Vertèbres totales (+CP1) & 39 & $39-40$ & 54 & $38-40$ & 17 & $38-40$ \\
\hline Vertèbres prédorsales & 9 & 10 & 54 & $9-10$ & 17 & $9-10$ \\
\hline Vertèbres postdorsales & 14 & $13-14$ & 53 & $13-15$ & 17 & $13-14$ \\
\hline Vertèbres anale - dorsale & 3 & $3-4$ & 53 & $3-5$ & 17 & $3-5$ \\
\hline Supraneuraux & 6 & 6 & 54 & $5-7$ & 17 & $6-7$ \\
\hline
\end{tabular}

Les serrae prépelviennes commencent à apparaître à partir de $22 \mathrm{~mm}$ LS. Leur nombre atteint 20 à 25 vers $50 \mathrm{~mm}$ de LS puis reste constant (Figure 6b). Le nombre total de serrae suit la même progression et varie entre 35 et 41 chez les spécimens de plus de $50 \mathrm{~mm}$ de LS. Les serrae postpelviennes apparaissent avant les serrae prépelviennes. Leur nombre se maintient entre 12 et 17 chez les spécimens de plus de $26 \mathrm{~mm}$ de LS, dont 6 à 10 serrae simples et 3 à 8 épines doubles de part et d'autre du cloaque. Les serrae prépelviennes, d'abord réduites à une épine, présentent une base formée de deux ailes dorsales courtes. Les serrae postpelviennes sont toujours plus hautes que les serrae prépelviennes. Le profil de la section préventral est arrondi et ne présente pas ou peu de carène médiane.

Les branchiospines sont allongées, coniques, et présentent une fine membrane lobulée sur la face interne. Leur nombre varie de 13 à 17 sur la rameau supérieur du premier arc branchial et de 15 à 18 sur le rameau inférieur chez les spécimens de plus de $40 \mathrm{~mm}$ de LS. 
Sur plus de 70 spécimens de 20 à $250 \mathrm{~mm}$ de LS, nous avons compté 38 à 40 vertèbres plus le centrum préural (CP1) le long du squelette axial dont 9-10 prédorsales et 13-15 (+ CP1) postdorsales. Nous avons aussi observé 5-7 supraneuraux en avant du premier ptérygiophore de la dorsale, et 3-5 vertèbres entre l'insertion du premier ptérygiophore de l'anale et l'insertion du dernier ptérygiophore de la dorsale. Aucune variation liée à la taille des individus n'a été observée pour ces caractères.

\section{Squelette}

\section{Les mâchoires}

Les dents sont fortement implantées sur les mâchoires.

Le prémaxillaire est laminaire, peu courbé (Figure 4). Le processus ascendant est fin, pointu à son extrémité distale et oblique par rapport au bord antérieur du processus latéral. Le processus latéral du prémaxillaire est sub-triangulaire et limité au niveau de la dernière dent chez les spécimens de moins de $150 \mathrm{~mm}$ de LS. Chez les spécimens plus longs, le prémaxillaire est sub-rectangulaire et la pointe dorso-postérieure est projetée légèrement en arrière de la dernière dent. Un processus transversal en forme de pointe est situé juste au-dessus de la dernière dent. En vue linguale, le processus ascendant présente une paroi osseuse fine et sagittale sur toute sa hauteur. Le processus latéral présente une fosse allongée pour les dents de remplacement au niveau des dents 2 à 4 . Une autre fosse plus arrondie et plus petite est située au-dessus de la dent 5 . Le maxillaire est réduit à une lame osseuse étroite. In situ, le maxillaire est articulé ventralement avec le processus transversal du prémaxillaire et dorsalement avec la projection dorsopostérieure du processus latéral du prémaxillaire. Chez les spécimens de 22 et $23 \mathrm{~mm}$ de LS, le maxillaire en vue latérale est plus large, en forme de goutte d'eau. L'examen de ces spécimens révèle aussi l'absence de dents coniques sur l'ectoptérygoïde.

Le dentaire est laminaire et allongé (Figure 5). La suture médiane est composée de 4 à 5 indentations chez les adultes. L'aire d'insertion des dents sur l'os est régulièrement et faiblement oblique. La fosse de remplacement des dents labiales est obstruée par une fine lame osseuse ponctuée d'une petite ouverture par dent, ces ouvertures étant parfois coalescentes. La fosse de remplacement de la dent symphysaire linguale est située immédiatement au-dessous de cette dent. Le processus dorso-postérieur se termine par une pointe projetée au-dessus et en arrière de l'angulo-articulaire. Sur la face linguale, le processus dorsal de l'angulo-articulaire forme un lobe allongé qui recouvre la moitié de l'aile dorsale du dentaire. Le bord de ce lobe est lisse. Les dents sont situées sur le bord labial de la lame osseuse du dentaire.

La position oblique de la bouche ne semble donc pas due à une morphologie particulière des os de la mâchoire.

\section{La série infra-orbitaire}

La suture entre l'infraorbitaire 1 (IO1) et l'infraorbitaire 2 (IO2) est très forte (Figure 9). L'IO2 est de forme triangulaire très marquée. L'IO3 est allongé et parcouru transversalement par un rameau du canal sensoriel branchu chez les grands spécimens. L'IO4 est de forme quandrangulaire et est parcouru par un rameau oblique du canal sensoriel. L'IO4 et l'IO5 sont parfois soudés chez les plus grands spécimens. L'IO6 est réduit et porte un canal sensoriel en Y. L'antéorbitaire a la forme d'une petite massue. Le supraorbitaire est large et proéminent au-dessus de l'œil. 


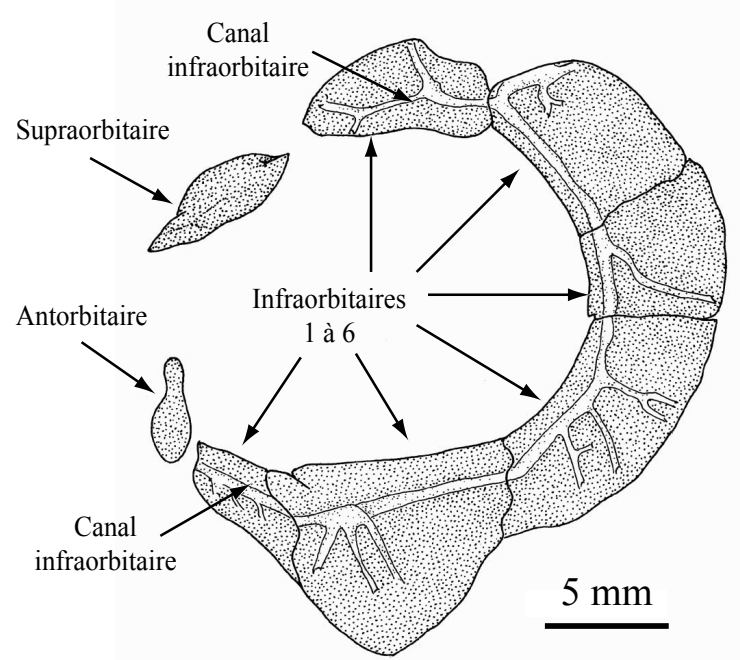

\section{Figure 9}

Tometes lebaili n. sp., MNHN 2001-1219, $155 \mathrm{~mm}$ de LS : os de la série infraorbitaire.

\section{Figure 9}

Tometes lebaili n. sp., MNHN 2001-1219, 155 mm SL: infraorbital bones serie.

\section{Le suspensorium}

Le processus antérieur de l'hyomandibulaire est pointu mais de taille réduite. La tête de l'hyomandibulaire est allongée et étroite. L'operculaire est étroit et allongé.

Le bord dorsal de l'ensemble méta-mésoptérygoïde est sinusoidal. Le bord postérodorsal du métaptérygoïde est anguleux, mais ne forme pas de pointe. En revanche, le bord postéro-ventral présente une encoche nettement marquée. Le mésoptérygoïde est triangulaire. L'ectoptérygoïde est réduit à une fine lame en demi-lune.

\section{L'arc hyoïde et l'appareil branchial.}

Les deux tiers proximaux des branchiospines, y compris la membrane postérieure, sont ornés de 6 à 10 séries de denticules coniques courbes. La forme de ces denticules est assez proche de celle des dents pharyngiennes, mais leur taille moyenne est de l'ordre du dixième de millimètre contre $0,5 \mathrm{~mm}$ pour les dents pharyngiennes.

La constriction médiane du cératohyal est très prononcée. L'interhyal est court et trapu.

\section{Le neurocrâne}

Le neurocrâne en vue latérale est de forme triangulaire, mais reste assez bas (Figure 10a). Le profil dorsal est rectiligne, sans concavité au niveau de la barre épyphyséale. Le profil du supraoccipital, en continuité avec le profil dorsal, est convexe en arrière de la suture pariétal-supraoccipital. L'aspect général du crâne est marqué par la taille importante de la fosse olfactive puis de la fosse orbitaire. En vue dorsale, le neurocrâne est assez massif, marqué par une importante fontanelle (Figure 10b). 

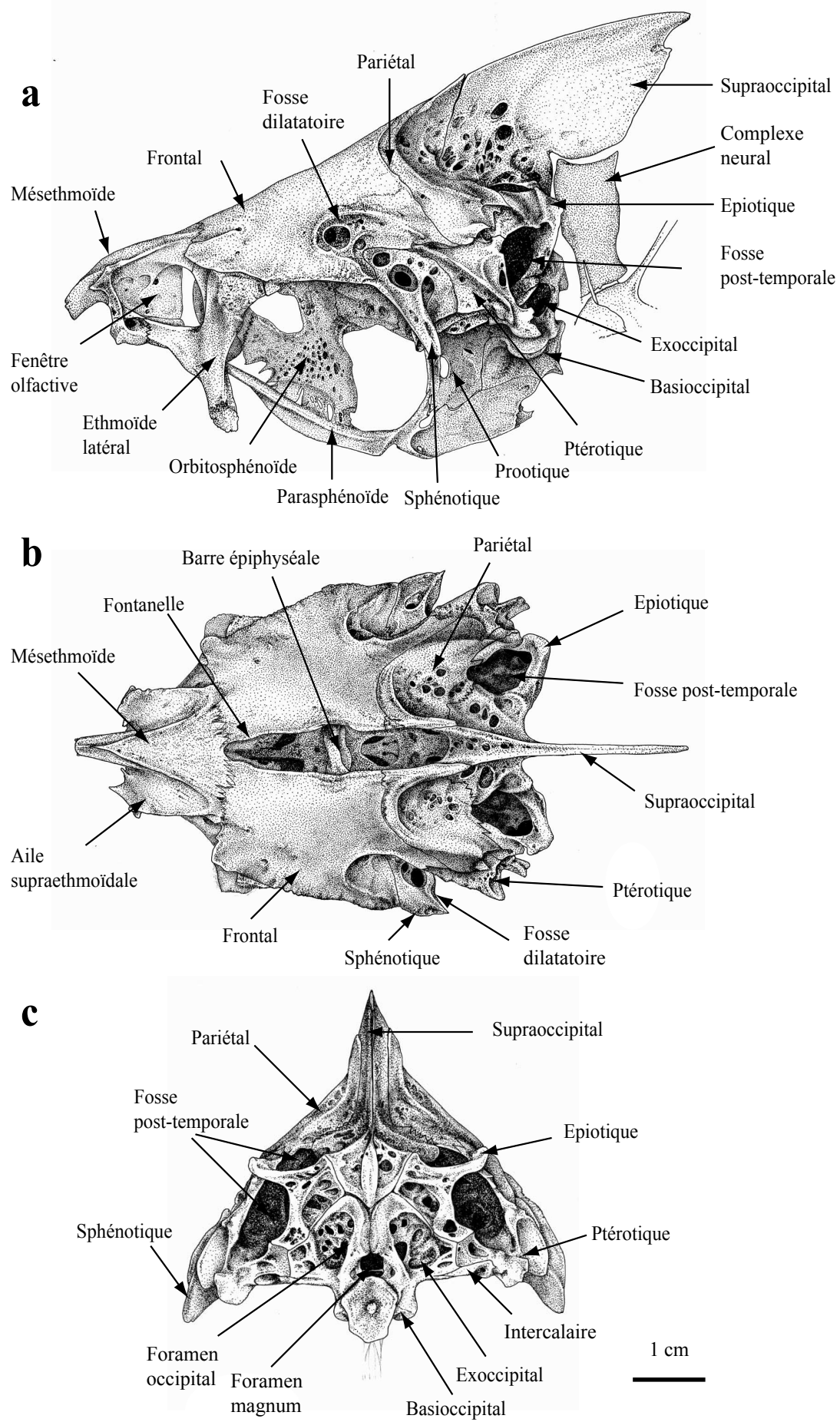

\section{Figure 10}

Tometes lebaili n. sp., MNHN 2001-1238, $300 \mathrm{~mm}$ de LS : neurocrâne en vue (a) latérale, (b) dorsale et (c) postérieure.

\section{Figure 10}

Tometes lebaili n. sp., MNHN 2001-1238, 300 mm SL: neurocrane in (a) lateral view, (b) dorsal view and (c) posterior view. 
La région ethmoïdienne. En vue dorsale (Figure 11c), le mésethmoïde est triangulaire avec de larges ailes supraethmoidales qui courent sur les 2/3 postérieur de l'os, atteignant presque la suture avec le frontal. Ces ailes déterminent toutefois des gorges d'insertion du prémaxillaire qui restent très peu profondes. Le mésethmoïde est projeté en avant du vomer en une pointe élancée.

En vue latérale, on note que cette pointe est incurvée vers le bas. La fenêtre olfactive est très largement ouverte (Figure 11a), limitée dorsalement par le toit du mésethmoïde qui est fin. Chez les adultes, le processus antérieur de l'ethmoïde latéral et le processus postérieur du mésethmoïde délimitent le bord ventral de la fenêtre dont est exclu le vomer qui vient se placer immédiatement sous la suture mésethmoïde-ethmoïde latéral. Le plancher de la fenêtre est situé plus bas que son bord, et il est recouvert d'une importante couche de graisse. Le septum internasal, qui sépare les capsules olfactives sur les deux tiers antérieurs, est une lame osseuse fine formée par une expansion dorsale du vomer et une expansion postéro-ventrale du mésethmoïde. Vers l'arrière, la fosse olfactive est limitée par une expansion médiane du latéral-ethmoïde semblable à celle observée chez Tometes trilobatus (JÉGU et al., sous presse : Figure 13a). Toutefois ces lames sont plus fortes chez $T$. lebaili et ne présentent pas de digitation latérale. Le foramen olfactif est largement ouvert et le canal du ramus ophthalmicus est bien visible en vue frontale.

En vue ventrale, la pointe du mésethmoïde est projetée bien en avant du vomer (Figure 11b). Le vomer est en forme d'écusson court prolongé vers l'arrière par un appendice ovaloïde dont la longueur est à peu près égale à la largeur de l'écusson. Le vomer ne couvre pas tout le plancher des fosses nasales, laissant des lacunes situées de part et d'autre du vomer jusqu'aux processus antérieurs de l'ethmoïde latéral. Un tissus osseux fin constitue le plancher des capsules olfactives à ce niveau (Figure 10b).

La région orbitaire. L'ethmoïde latéral, le bord latéral du frontal et la pointe du sphénotique déterminent une ample cavité orbitaire dont la longueur atteint plus de $40 \%$ de la longueur du neurocrâne. La pointe du sphénotique est située bien en arrière du niveau de la barre épiphyséale.

L'orbitosphénoïde forme un « $L$ » inversé. La suture avec le frontal est fortement digitée et oblique jusqu'au toit de l'orbite. Vers l'avant du processus ascendant de l'orbitosphénoïde et du processus descendant du frontal, une fine lame osseuse forme le toit du myodome antérieur, alors que la limite ventro-latérale est déterminée par le processus médian de l'ethmoïde latéral. Le processus antérieur de l'orbitosphénoïde atteint la parasphénoïde chez les grands spécimens, formant une suture forte. Le processus antérieur de l'orbitosphénoïde vient au contact du processus médian de l'ethmoïde latéral. II se prolonge vers l'avant par une fine lame osseuse verticale qui court le long du bord ventral du processus médian de l'ethmoïde latéral, isolant les branches droite et gauche du myodome antérieur.

Le foramen optique est très amplement ouvert, triangulaire, limité vers l'avant par l'orbitosphénoïde, latéralement par le ptérosphénoïde et postérieurement par le prootique.

Le ptérosphénoïde est de forme générale hexagonale et présente de nombreux foramens dont celui du nerf trochléaire, complètement inclus dans l'os (Figure 12a). Cet os présente une surface courbe homogène, orienté antéro-ventralement.

En vue frontale, le processus épineux du sphénotique est en forme d'aile. Le bord ventral forme une courbe continue avec le bord du prootique, avec toutefois une forte convexité au niveau de la suture sphénotique-prootique. La face frontale du sphénotique est marquée par de nombreux foramens dont certains sont directement ouverts sur la fosse dilatatoire. 

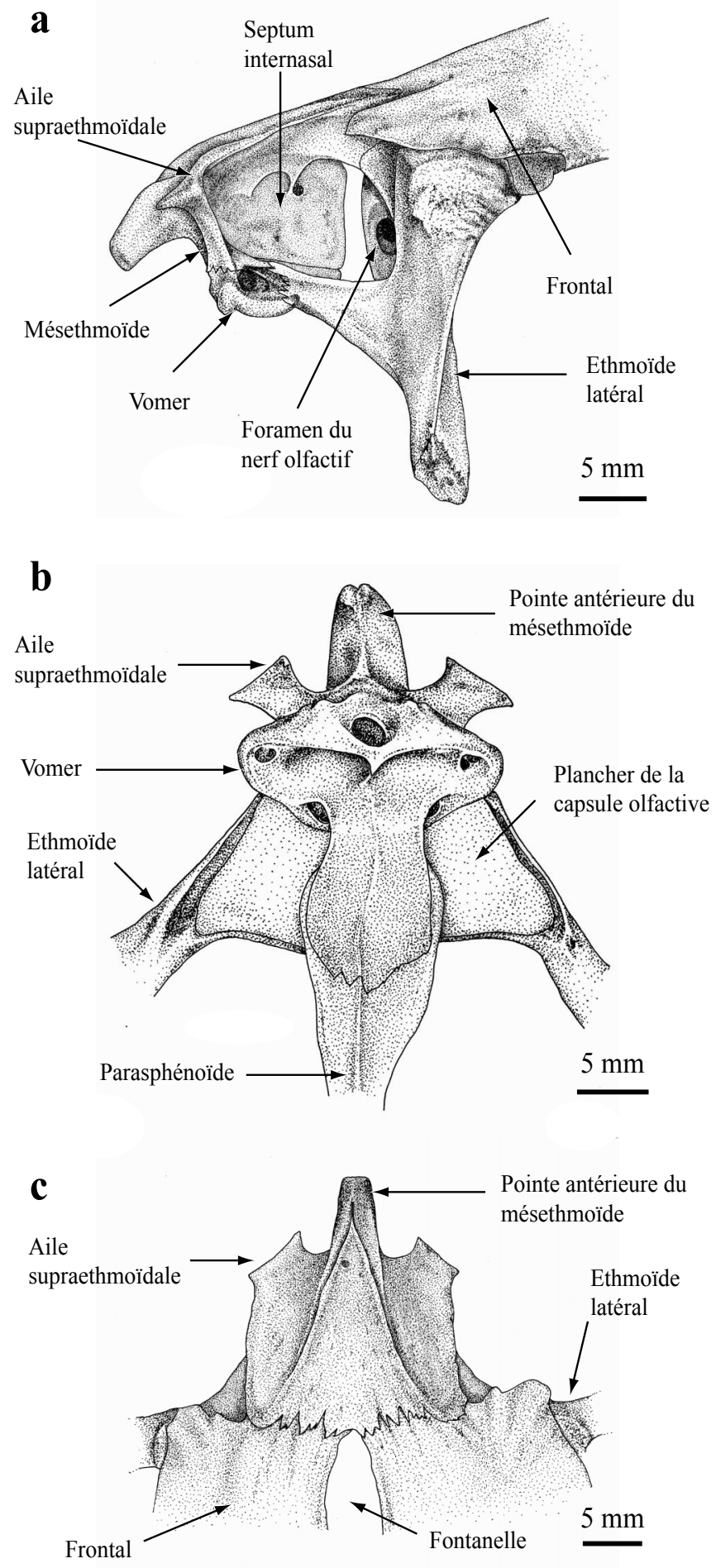

\section{Figure 11}

Tometes lebaili n. sp., MNHN 2001-1238, $300 \mathrm{~mm}$ de LS, région ethmoidienne : (a) vue latérale gauche, (b) vue ventrale et (c) vue dorsale.

\section{Figure 11}

Tometes lebaili n. sp., MNHN 2001-1238, $300 \mathrm{~mm}$ SL, ethmoidian area: (a) left lateral view, (b) ventral view and (c) dorsal view. 

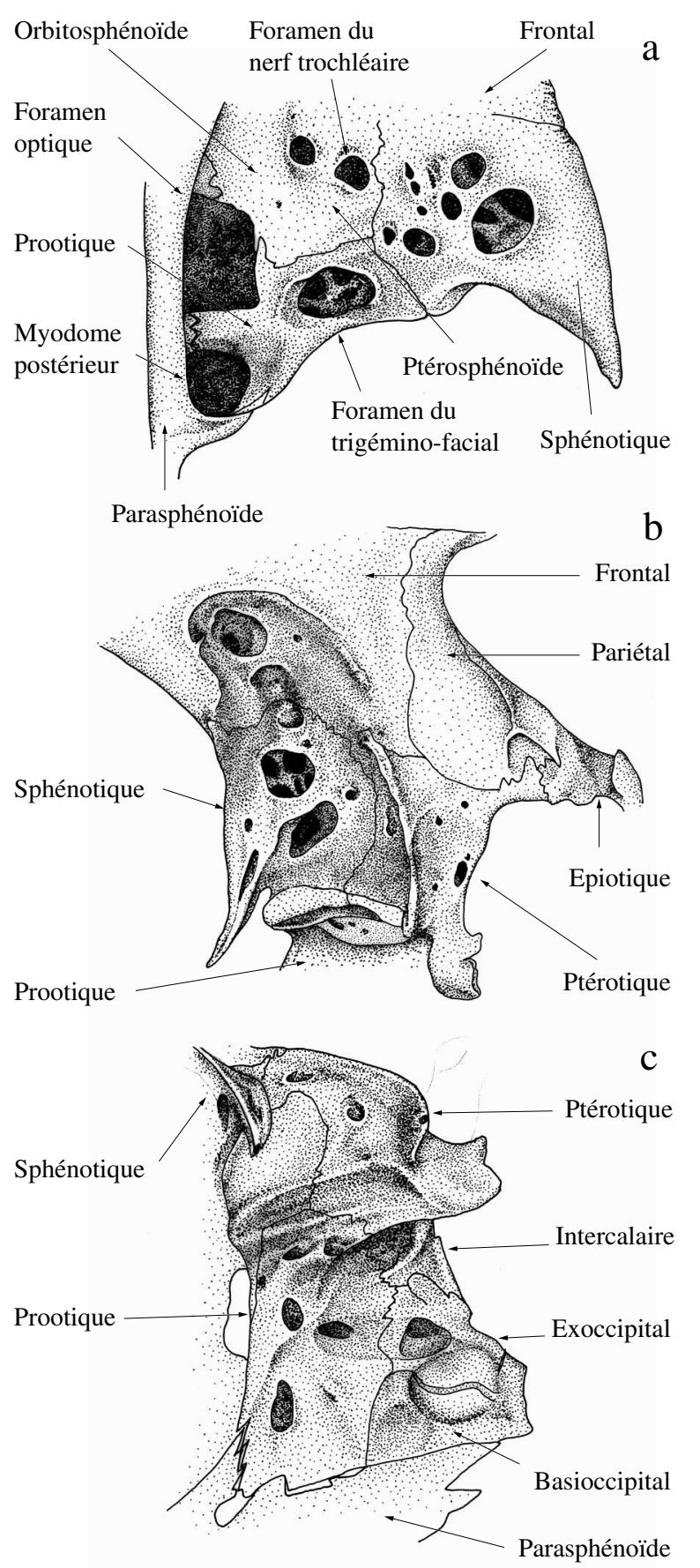

\section{Figure 12}

Tometes lebaili n. sp., MNHN 2001-1238, $300 \mathrm{~mm}$ de LS : (a) vue ventrale du toit orbitaire, (b) vue latérale de la fosse dilatatoire gauche et (c) vue latérale du prootique gauche.

\section{Figure 12}

Tometes lebaili n. sp., MNHN 2001-1238, $300 \mathrm{~mm}$ SL: (a) ventral view of the orbital roof, (b) lateral view of the left dilatatory fossae and (c) lateral view of the left prootic. 
La face frontale du prootique est sub-quadrangulaire et le foramen trigéminofacial est situé dans une légère dépression au centre de l'os. Le pont prootique qui sépare le foramen optique du myodome postérieur est situé sur le même plan vertical que le bord antérieur du prootique. Le myodome postérieur est très largement ouvert mais sur un seul compartiment et jusqu'au bord postérieur du parasphénoïde. Le muscle droit externe de l'œil occupe seul le myodome postérieur.

La fosse dilatatoire et la région otique. La fosse dilatatoire est allongée, profonde et étroite (Figure 12b). La face latérale du sphénotique, qui détermine la partie ventrale de la fosse, est de forme losangique à grand axe vertical. Le bord postéro-ventral de la fosse est nettement marqué par une lame verticale du ptérotique alors que le bord postérodorsal, sur le frontal, est peu marqué. La suture frontal-sphénotique dans la fosse dilatatoire est légèrement décalée en regard de cette suture dans la cavité orbitaire, les deux os présentant un léger recouvrement. La fosse dilatatoire est marquée par de nombreux foramens plus nombreux au niveau du sphénotique que du frontal. La base de l'épine sphénotique présente un pilier déterminé par un foramen de part et d'autre. Ces deux foramens sont ouverts sur un troisième foramen situé sous le pilier et lui-même directement ouvert sur la cavité orbitaire. C'est ce pilier qui marque la limite ventrale de l'insertion du dilatator operculi et la limite dorsale de l'insertion du levator arcus palatini suivant le même schéma que celui observé chez Tometes trilobatus (JÉGU et al., sous presse).

Dans la partie ventrale de la fosse, la suture sphénotique-ptérotique est en position médiane. Le processus postéro-ventral du ptérotique est réduit à une lame osseuse courte, divisée en une branche dorsale droite et une branche ventrale en forme de sagaie. La face latérale du ptérotique est étroite et allongée verticalement.

L'articulation de l'hyomandibulaire est courte en raison de l'extension réduite de l'ensemble de la fosse dilatatoire.

En vue latérale, le prootique est de forme rectangulaire, plus haut que large (Figure 12c). Le foramen auditif est plus large que le foramen du tronc hyomandibulaire du nerf facial situé immédiatement devant. La suture parasphénoïde-prootique d'abord digitée est ensuite rectiligne, tout comme l'articulation prootique-basioccipital. La suture prootiqueptérotique est inclue dans la fosse temporale. Celle-ci est bordée ventralement par l'intercalaire, en forme de « $T$ 》 couché, bombé et fermement soudé à l'exoccipital. La face latérale de l'exoccipital est triangulaire. Le foramen du dixième nerf cranial (vagus) est large et situé juste au-dessus de la bulle osseuse renfermant l'astericus. Le bord ventral du basioccipital est rectiligne et la suture parasphénoïde-basioccipital est droite, située dans l'alignement de la suture avec le prootique.

Région occipitale. L'aire située entre la fosse dilatatoire et le canal supratemporal est très réduite. La branche du frontal et la partie du pariétal située en avant du canal supratemporal sont très étroites (Figure 10a). En revanche, en arrière du canal supratemporal, l'os pariétal forme une large plage. La zone située en arrière du canal supratemporal forme une dépression dont le bord antérieur ne forme pas de repli pouvant constituer le toit d'une cavité comme on peut l'observer chez Colossoma. La base du supraocciptal et la partie du pariétal formant cette plage en arrière du canal supratemporal sont formées de tissus osseux hautement réticulé.

La fosse post-temporale dorsale est sub-triangulaire, limitée à l'arrière par la branche médiane de l'épiotique qui forme la base du triangle. La branche de l'épiotique est réduite à une tige osseuse fine et la suture supraoccipital-épiotique forme l'angle postéroproximal de la fosse.

En vue postérieure, la fosse post-temporale ventrale est large et oblique (Figure 10c). En raison de la proximité de la fosse dilatatoire, l'expansion du ptérotique, 
limite postérieure de la fosse dilatatoire, dessine une aile latéro-ventrale large sur laquelle vient se superposer le processus latéro-postérieur du ptérotique. La pointe postéroventrale du supra-occipital est située ventralement par rapport à la barre transversale de l'épiotique. L'exoccipital est un os réticulé dont les sutures avec l'épiotique et le supraoccipital sont simples et rectilignes, alors que la suture exoccipital-intercalaire est très digitée. Le foramen occipital, sub-oval et vertical, est situé au centre de l'os. Comme chez T. trilobatus, on observe un petit foramen pair en position distale par rapport au cavum sinus imparfinis et au foramen magnun. Au contact avec le scaphium, ce foramen est directement ouvert dans la cavité céphalique.

En vue latérale, le supraoccipital est court et son profil dorsal est convexe. Le profil ventral du supraoccipital est composé d'une articulation courte avec le complexe neural, et d'une partie oblique rectiligne jusque la pointe dorso-postérieure.

\section{L'appareil de Weber}

Le complexe supraneural est subrectangulaire, étroit et allongé verticalement (Figure 10a). Le bord postéro-ventral du complexe neural n'entre pas en contact avec la neurapophyse de la quatrième vertèbre. Le processus ascendant de l'arc neural de la troisième vertèbre est court (environ $20 \%$ de la hauteur du complexe neural) et situé en arrière de la projection latérale du complexe neural. Le processus latéral de la seconde vertèbre est allongé et de section circulaire.

Les côtes pleurales 5 , et dans une moindre mesure les côtes pleurales 6 , développent un processus postéro-médian triangulaire qui vient s'appuyer sur la côte suivante formant un ensemble sur lequel s'appuie la chambre antérieure de la vessie natatoire.

\section{Le squelette appendiculaire}

Ceintures pectorale et pelvienne. A la ceinture pectorale, le premier post-cleithrum est séparé du second. Le post-cleithrum 2 est allongé. Le processus médio-postérieur du post-cleithrum 3 est rectangulaire. La pointe ventrale du post-cleithrum est allongée et dépasse le bord ventral du coracoïde in situ. Le bord postérieur du cleithrum est ventru et lisse, sans épine antrorse à l'extrémité ventrale du processus latéral transverse. Ce processus forme un repli marqué au-dessus de la surface du cleithrum. Le bord antérieur du cleithrum est régulièrement concave, sauf au niveau de l'attache du mésocoracoïde où il est légèrement convexe. L'attache du mésocoracoïde au cleithrum est formée d'un processus dorsal arrondi et d'un processus postérieur fin. L'attache de la scapula forme un processus dorso-ventral effilé. En vue latérale interne, la suture scapula-mésocoracoïdecoracoïde est située au niveau médian du processus ascendant du coracoïde. Le coracoïde est court et haut. Le processus antérieur du coracoïde est court et son articulation avec le cleithrum est étroite. La fenêtre coracoïde-cleithrum est réduite. Le bord ventral du coracoïde est régulièrement convexe, alors que le bord postéro-dorsal se termine en pointe. Chez les spécimens de moins de $30 \mathrm{~mm}$ de LS, la pointe est située sur le bord postéro-ventral du coracoïde.

A la ceinture pelvienne, la longueur du processus ischiatique atteint 55 à $60 \%$ de la longueur de l'os pelvien. Le processus ischiatique est laminaire, fin et rectiligne.

Squelette des nageoires dorsale et caudale. L'épine prédorsale est pointue et allongée. Le bord antéro-dorsal du ptérygiophore est régulièrement convexe.

Le squelette caudal est d'une configuration très proche de celle de T. trilobatus (JÉGU et al., sous presse : Figure 17). En particulier, l'hypural II est libre de l'hypural I et du centrum préural (CP1). L'hypural III, soudé au CP1 est réduit à une bande osseuse étroite. La pointe antérieure de l'hypural IV est accolée au CP1. 


\section{Coloration}

\section{Spécimens préservés en alcool}

Les spécimens de moins de $20 \mathrm{~mm}$ LS présentent un corps marbré de taches sombres sur fond plus clair (Figure 2). En avant de la dorsale, on observe 2 à 3 plages sombres, dont une au-dessus des yeux et qui peuvent descendre jusqu'à la moitié des flancs. Une tache plus large, située au niveau de l'origine de la base de la dorsale, s'étend plus bas que la ligne latérale sur les flancs et sur le premier tiers des premiers rayons de la dorsale. Deux à trois taches moins hautes sont situées le long de la base de la dorsale et au niveau de l'adipeuse. Au niveau de la ligne latérale, une à deux taches rondes sont situées en avant de la grande tache verticale. En arrière, une grosse tache plus sombre est suivie par une bande foncée qui s'étend jusqu'à la base de la caudale. Cette ensemble de taches médianes prend l'aspect d'une barre longitudinale irrégulière, avec un pseudoocelle plus foncé juste sous la dorsale. En-dessous de la ligne latérale, on observe 3 à 4 bandes verticales irrégulières en avant de l'anale, puis 3 à 4 taches rondes situées au niveau de la base de l'anale. Une barre verticale ténue marque la fin de l'hypural.

Les premiers rayons de la dorsale sont noirs sur le tiers basal et cette tache se prolonge le long des trois premiers rayons en un liseré parfois bien marqué. Les premiers rayons de l'anale sont aussi grisés. Le reste des nageoires ne présente pas de coloration.

Entre 20 et $50 \mathrm{~mm}$ LS, on retrouve le même patron de coloration sur les flancs mais déjà plus atténué au-delà de $40 \mathrm{~mm}$ LS. A partir de $30 \mathrm{~mm}$ LS, non seulement le bord antérieur des nageoires anale et dorsale présente une bande noire, mais le bord distal de ces nageoires est aussi marqué par une bande noire. Le bord distal de la caudale présente aussi un liseré noir. Aucun spot huméral noir n'a été observé chez ces individus.

Chez les spécimens de 100 à $130 \mathrm{~mm}$ de LS, le corps est brun clair avec des plages plus sombres (Figure 2a). Sur un individu de $110 \mathrm{~mm}$ de LS, on note la présence très ténue d'une tache humérale ronde, de la taille de l'œil, située sur la ligne latérale entre les écailles 6 et 10. Le bord libre des nageoires dorsale, anale et caudale présente une bande noire. Sur la caudale, cette bande noire est plus large et nettement délimitée. La moitié distale des rayons qui forment le lobe anal antérieur est noire. La pointe du lobe dorsal est noire. Le premier rayon des pectorales est sombre et un liseré noir borde l'adipeuse.

Les grands spécimens sont très foncés à noirs, les nageoires étant généralement plus noires que les flancs.

\section{Spécimens vivants}

Les flancs des grands spécimens sont uniformément gris foncé à noirs (Figure 3). Les nageoires pectorales, pelviennes, dorsale, caudale et anale, le dessus de la tête et l'opercule sont noirs, avec parfois des reflets jaunes et noirs. Chez un mâle d'environ $400 \mathrm{~mm}$ de LS photographié de la Mana, on observe une coloration jaune-rouge sur les plages plus claires.

Les individus de 100 à $150 \mathrm{~mm}$ environ ont les flancs argentés avec de larges plages gris-noir. La tache humérale ronde observée sur l'individu préservé apparaît très nettement sur le gris argenté des flancs. La bande noire de la caudale couvre la moitié distale de la nageoire. Dorsale, anale et adipeuse sont presque complètement noires. Les nageoires pectorales et pelviennes restent claires.

\section{Biotope et distribution}

Les spécimens de la Mana ont tous été capturés dans des rapides, à la flèche ou à la ligne en appâtant avec des Podostemaceae. La plupart des spécimens du Haut 
Maroni, et en particulier ceux qui ont été capturés au cours de pêches traditionnelles à la roténone à Pierkuru dans le Tampok et sur l'Oulemari (PAGÉZY et JÉGU, 2002), ont aussi été capturés dans les rapides à Podostemaceae ou dans leur zone d'influence. Des observations préliminaires semblent indiquer que le régime alimentaire de $T$. lebaili, comme celui de T. trilobatus Valenciennes (JÉGU et al., sous presse), est principalement constitué de Podostemaceae, ces plantes rupestres abondantes dans les rapides du plateau Guyanais. Le régime alimentaire ne serait donc pas affecté par la différence observée dans la position de la bouche chez ces deux Tometes. Par ailleurs, SANTOS et al. (1997) ont montré que Mylesinus paraschomburgki, une autre espèce rhéophile du bouclier guyanais dont la dentition est très proche de celle de Tometes (JÉGU et al., 1989), s'alimentait exclusivement de Podostemaceae à l'état adulte. La longueur de l'intestin et le faible taux de méthyl-mercure (FRÉRY et al., 2001) rencontrés chez T. lebaili confirment l'origine végétale de son alimentation. L'hyperinfestation par les nématodes dans le tube digestif, qui est sans impact apparent sur l'état de santé des individus, rappelle un commensalisme.

En 1993, à l'INRA de Kourou (Guyane fr.), Mr. Planquette nous a affirmé que le spécimen MNHN 1998-304 avait été capturé par M. Lachenaud, dans le bas Sinnamary. Plusieurs années plus tard, Mr. Lachenaud (CIRAD, Montpellier, lettre du 09 janvier 1997) ne reconnaît pas dans les photos du MNHN 1998-304 les " gros coumarous " qu'il avait capturés à Pointe Combi et qui en différeraient par " la tête, l'œil et le nombre de dents ". Aucun autre individu n'ayant été capturé sur le Sinnamary au cours des nombreuses collectes réalisées par I'INRA et I'IRD (ex-ORSTOM) dans le cadre des études préliminaires sur le barrage de Petit Saut, ni depuis sa fermeture dans les collectes de routine, un doute subsiste sur l'origine de ce spécimen. En revanche, la présence de Tometes lebaili est confirmée dans la Mana, le bassin du Maroni et plus à l'ouest dans la Commewine au Surinam, un bassin en liaison avec le Maroni par la rivière Cottica. II semble donc que cette espèce remplace Tometes trilobatus présent du bassin de l'Araguari à celui de l'Oyapock (JÉGU et al., sous presse).

\section{DISCUSSION}

Tometes lebaili partage la plupart des caractères morpho-anatomiques de Tometes décrits par JÉGU et al. (sous presse) pour T. trilobatus, espèce-type du genre.

T. lebaili diffère ainsi de Mylesinus par la forme sigmoïde et courte des dernières dents du prémaxillaire (incisives et planes chez Mylesinus), la position relative des dents de la série linguale, accolées latéralement en arrière de la série labiale (séparées l'une de l'autre et insérées entre les dents de la série labiale chez Mylesinus) et le processus médian du prémaxillaire réduit à un appendice chez $T$. lebaili mais développé en un processus laminaire large chez Mylesinus. T. lebaili diffère aussi de Mylesinus par un nombre de dents plus faible au dentaire (6-7 contre 7-14 chez Mylesinus) et un plus grand nombre de serrae en avant des pelviennes (19-26 contre moins de 12 chez Mylesinus).

T. lebaili se différencie de $T$. trilobatus par la position de la bouche tournée vers le haut mais aussi par un plus grand nombre de dents au dentaire (6-7 vs. 5 pour T. trilobatus) et par un plus grand nombre de serrae en avant des nageoires pelviennes (20-26 vs. 25-28 pour T. trilobatus). Nous avons pu noter que, chez T. lebaili, les gorges formées par les ailes supraethmoidales sont étendues sur les deux derniers tiers du mésethmoïde, jusqu'à la suture avec le frontal. Chez $T$. trilobatus et les espèces des genres proches, comme Myleus setiger ou Mylesinus paraschomburgkii, elles sont limitées au second tiers (JÉGU et SANTOS, 2002 ; JÉGU et al., sous presse). Toutefois, le processus ascendant du prémaxillaire chez $T$. lebaili n'est pas différent de celui que l'on observe chez T. trilobatus. La gorge supraethmoïdale plus profonde permet un glissement vers l'arrière du prémaxillaire dont le processus latéral prend alors une position oblique qui se traduit par la forme de la bouche, oblique tournée vers le haut. L'allongement des ailes 
supraethmoidales vers l'arrière doit donc être considéré comme une autapomorphie pour Tometes lebaili.

Tometes trilobatus et $T$. lebaili sont des espèces importantes pour les amérindiens Wayãpi (OUHOUD-RENOUX, 1998 ; ROMEU, 2000) et Wayana (PAGÉZY et JÉGU, 2002). Pourtant, la revalidation de Tometes trilobatus et la description de T. lebaili n'ont pu être réalisées que grâce à de récentes collectes dans les milieux difficiles d'accès que sont les rapides. S'agissant d'espèces sur lesquelles les pressions anthropiques ne cessent de croître (pêche, destruction des biotopes par la prospection minière et les barrages), l'élargissement vers l'ouest des inventaires de ces milieux s'avère nécessaire.

\section{ÉTYMOLOGIE}

Tometes lebaili est dédié au Dr. P.Y. Le Bail, INRA, Rennes en hommage à l'important travail de collectes et de révisions des poissons de Guyane française qu'il a déjà réalisé. Le Dr. Le Bail est aussi le premier scientifique à avoir collecté $T$. lebaili dont il n'a pu rapporter que des photographies tant cette espèce est savoureuse et appréciée.

\section{REMERCIEMENTS}

Nous remercions Mme Beaunier, le Dr. Meunier et le Dr. Le Bail pour la relecture du manuscrit, leurs suggestions et les informations additionnelles qu'ils ont eut la gentillesse de nous indiquer. Nos remerciements particuliers au regretté Paul Planquette qui n'a jamais ménagé ses efforts pour rapporter et mettre à notre disposition les premiers spécimens du Haut Maroni. Nos remerciements aux Wayana du haut Maroni et au Dr. Y. Fermon pour leur aide précieuse dans notre quête du watau yaikë. Les dessins sont de E. Belmont-Jégu.

\section{RÉFÉRENCES}

COURTEMANCHE M., LEGENDRE V., 1985. Os de poissons : nomenclature codifiée, noms anglais et français. Bibliothèque nationale du Québec, $61 \mathrm{p}$.

CUVIER G., VALENCIENNES A., 1850. Histoire Naturelle des Poissons, 22, i-xx, 1-532.

FINK W.L., 1993. Revision of the piranha genus Pygocentrus (Teleostei, Characiformes). Copeia, 1993 (3), 665-687.

FRÉRY N., MAURY-BRACHET R., MAILLOT E., DEHEEGER M., MÉRONA B. DE, BOUDOU A., 2001. Gold-mining activities and Mercury Contamination of Native Amerindian Communities in French Guiana: Key role of fish in dietary Uptake. Environm. Health Persp., 109 (5), 449-456.

GÉRY J., PLANQUETTE P., LE BAIL P.Y., 1991. Faune characoïde (poissons ostaryophysaires) de l'Oyapock, l'Approuague et la rivière de Kaw (Guyane française). Cybium, 15 (1), 1-69.

JÉGU M., LEÃO E.L.M., SANTOS G.M. DOS, 1991. Serrasalmus compressus, une espèce nouvelle du Rio Madeira, Amazonie (Pisces, Serrasalmidae). Ichthyol. Explor. Fresh., 2 (2), 97-108.

JÉGU M., SANTOS G.M. DOS, 2002. Révision du statut de Myleus setiger Müller et Troschel, 1844 et de Myleus knerii (Steindachner, 1881) (Teleostei : Characidae : Serrasalminae) avec une description complémentaire des deux espèces. Cybium, 26 (1), 33-57.

JÉGU M., SANTOS G.M. DOS, FERREIRA E., 1989. Une nouvelle espèce du genre Mylesinus (Pisces, Characiformes), M. paraschomburgkii, décrite du rio Uatumã et du rio Trombetas (Brésil, Amazonie). Revue Hydrobiologie Tropicale, 22 (1), 49-62. 
JÉGU M., SANTOS G.M. DOS, KEITH P., LE BAIL P.Y., sous presse. Description complémentaire et revalidation de Tometes trilobatus Valenciennes, 1850, espècetype de Tometes Valenciennes (Teleostei : Characidae : Serrasalminae), Cybium, 26.

KEITH P., LE BAIL P.Y., PLANQUETTE P., 2000. Atlas des Poissons d'Eau douce de Guyane. Tome 2 fascicule I. Batrachoidiformes, Mugiliformes, Beloniformes, Cyprinodontiformes, Synbranchiformes, Perciformes, Pleuronectiformes, Tetraodontiformes. Patrimoines naturels, 43 (1), $286 \mathrm{p}$.

LE BAIL P.Y., PLANQUETTE P., GÉRY J., 1984. Clé de détermination des poissons continentaux et côtiers de Guyane. IV. Familles généralement inféodées au milieu dulçaquicole non Siluriformes. Bulletin de liaison du GRAAG, Kourou, 9, 1-97.

LEITE R.G., JÉGU M., 1990. Régime alimentaire de deux espèces d'Acnodon (Characiformes, Serrasalmidae) et habitudes lépidophages de $A$. normani. Cybium, 14 (4), 353-359.

MERCKX A., JÉGU M., SANTOS G.M. DOS, 2000. Une nouvelle espèce de Serrasalmus (Teleostei : Characidae : Serrasalminae), S. altispinis n. sp., décrite du rio Uatumã (Amazonas, Brésil) avec une description complémentaire de $S$. rhombeus (Linnaeus, 1766) du plateau Guyanais. Cybium, 24 (2), 181-201.

OUHOUD-RENOUX F., 1998. De l'outil à la prédation. Technologie culturelle et ethnoécologie chez les Wayãpi du haut Oyapock (Guyane française). Thèse présentée en vue du grade de Docteur à l'Université de Paris X, $455 \mathrm{p}$.

PAGÉZY H., JÉGU M., 2002. Valeur patrimoniale de Serrasalminae herbivores du Haut Maroni. Approches biologique et socio-culturelle. Bull. Fr. Pêche Piscic., 364, 49-69.

PLANQUETTE P., KEITH P., LE BAIL P.Y., 1996. Atlas des Poissons d'Eau douce de Guyane (tome 1). Patrimoines Naturels, 22, $429 \mathrm{p}$.

ROMEU S., 2000. L'enfant Wayãpi et la rivière. Exploitation des ressources naturelles accessibles par le cours d'eau. Mémoire de DEA Environnement : temps, espace, société, Université d'Orléans, 165 p.

SANTOS G.M., PINTO S.S., JÉGU M., 1997. Alimentação do pacu-cana Mylesinus paraschomburgkii (Teleostei, Serrasalmidae) em rios da Amazônia brasileira. Rev. Brasil. Biol., 57 (2), 311-315.

TAYLOR W.R., VAN DYKE G.C., 1985. Revised procedures for staining and clearing small fishes and other vertebrates for bone and cartilage study. Cybium, 9 (2), 107-119.

VALENCIENNES A., 1850. Voir CUVIER et VALENCIENNES. 